\title{
Abstracts of the 6th International Workshop on the CCN Family of Genes
}

\author{
Held at the Slieve Donnard Hotel, Newcastle, Northern Ireland \\ 20-24 October 2010
}

\section{HORMESIS IS CENTRAL TO TOXICOLOGY, PHARMACOLOGY AND RISK ASSESSMENT \\ 1-Calabrese Edward}

This presentation summarizes numerous conceptual and experimental advances over the past two decades in the study of hormesis. Hormesis is now generally accepted as a real and reproducible biological phenomenon, being highly generalized and independent of biological model, endpoint measured and chemical class/physical stressor. The quantitative features of the hormetic dose response are generally highly consistent, regardless of the model and mechanism and represents a quantitative index of biological plasticity at multiple levels of biological organization. The hormetic dose response model has been demonstrated to make far more accurate predictions of responses in low dose zones than either the threshold or linear at low dose models. Numerous therapeutic agents widely used by humans are based on the hormetic dose response and its low dose stimulatory characteristics. It is expected that as low dose responses come to dominate toxicological research that risk assessment practices will incorporate hormetic concepts in the standard setting process.

\section{BYSTANDER EFFECTS AND ADAPTIVE RESPONSES INDUCED BY RADIATION EXPOSURE}

\section{Carmel Mothersill}

McMaster University, Hamilton, Ontario, Canada

This presentation reviews our current knowledge of the mechanisms underlying the induction of bystander signaling effects by low doses ionizing radiation and discusses how they may be related to observed adaptive responses or other protective/ hormetic effects of low doses exposures. Bystander effects appear to be the result of a generalized stress response in tissues or cells. The signals may be produced by all exposed cells but the response appears to require a quoram in order to be expressed. The major response involving low LET radiation exposure discussed in the existing literature is a death response. This has many characteristics of apoptosis. Signal production by the irradiated cells is now known to involve very different mechanisms which can be independently modulated. Signal production is clearly triggered by deposition of radiation energy and our current hypothesis is that this affects cell membranes much like a nerve impulse. We have evidence that low doses cause serotonin to bind to the membrane opening a specific 5HT $3 \mathrm{~A}$ calcium channel. The transient influx of calcium triggers downstream events resulting in release of ROS, NOS and possibly TNF alpha from the targeted cells. The recipients of the signal molecules induce apoptosis in our system but in other genetic backgrounds they appear to produce growth responses. While a death signal and response might appear to be adverse, the position is argued in this paper, that it is in fact protective and removes damaged cells from the population. Since many cell populations carry damaged cells without being exposed to radiation (so called "background damage", it is possible that low doses exposures cause removal of cells damaged by agents other than the test dose of radiation. This mechanism would lead to the production of "U-shaped" dose response curves. In this scenario, the level of "adaptive" or beneficial response will be related to the background damage carried by the cell population. This model may be important when attempting to predict the consequences of mixed exposures involving radiation and other environmental stressors. It also has practical benefits as it could suggest new therapeutic options where control of stress signaling and response is needed.

\section{ANGIOGENESIS INHIBITION COMES UNDONE}

Andrew R. Reynolds

Breakthrough Breast Cancer Research Centre, The Institute of Cancer Research, 237 Fulham Road, London SW3 6JB, UK

Tumour angiogenesis, the growth of new blood vessels into tumours, facilitates tumour growth and thus represents an attractive therapeutic target for the treatment of cancer. Numerous angiogenesis inhibitors have been trialled in patients. These include drugs that target cell surface adhesion molecules expressed on tumour blood vessels, e.g. integrin inhibitors, and drugs that target pro-angiogenic growth factors, e.g. vascular endothelial growth factor (VEGF) inhibitors. Some of these agents have failed to show any substantial activity in patients (e.g. integrin inhibitors) whilst others, although they show activity in a subgroup of patients, provide only a few months increment in patient survival due the presence of acquired resistance (e.g. VEGF inhibitors). Recent 
work has focused on understanding the effects of angiogenesis inhibitors on tumour biology and has revealed a number of new findings that may help to explain resistance to these drugs. For example, we have shown that low (nanomolar) concentrations of integrin inhibitors can stimulate tumour growth and tumour angiogenesis in mice, whilst inhibition is observed only at micromolar doses i.e. a bell-shaped dose-response is observed (Reynolds et al., 2009 Nat. Med. 15(4):392-400). Moreover, it appears that numerous other angiogenesis inhibitors can also display bellshaped or U-shaped dose-response curves (Reynolds 2010, Dose Response 8(3):253-284). These unexpected dose-response phenomena may limit significantly the potential for these drugs to be used in the clinic. Interestingly, recent work from other laboratories has shown that VEGF inhibitors can accelerate the growth of tumour metastases in mice, sparking concern for the use of these drugs in patients (Paez-Ribes et al., 2009 Cancer Cell 15(3):220-31, Ebos et al., Cancer Cell 15(3):232-239). We have recently examined this phenomenon in mice and found that inhibition of the VEGF pathway can either suppress or promote tumour progression, depending on the tumour model utilised. We are currently using these tumour models in order to understand how these drugs can exert opposing effects on tumour progression. The eventual goal of this work is to understand how the administration of anti-angiogenic drugs to patients can be optimised for the greatest therapeutic benefit.

\section{LOW-DOSE IONIZING RADIATION INDUCES SIGNALING TOWARDS PROTECTION AND HORMESIS}

Ludwig E. Feinendegen, M.D.

Progessor eneritus, Heinrich-Heine University Düsseldorf, Germany and Guest Scientist, Brookhaven National Laboratory, Upton, NY, USA

Ionizing radiation deposits energy through subatomic charged particle tracks that cause atomic-molecular ionizations and excitations along the tracks in a stochastic manner in the exposed tissue. Besides direct hits on vital molecules there are stochastic interactions with reactive oxygen species (ROS) from radiolysis of water that constitutes about $80 \%$ of soft tissue mass. Thus, an average Compton electron track of $\sim 6 \mathrm{keV}$ from 100 $\mathrm{kVp}$ x-rays causes per hit ng mass (cell), amounting to an absorbed dose of $\sim 1 \mathrm{mGy}$, a burst of $\sim 150$ ROS. Low-dose irradiation means single or few tracks per hit cell, either from acute or protracted exposure. Radiationinduced cell damage may be severe enough to affect the entire body.

Mammalian cells react promptly and very sensitively to very low doses by activating signal pathways, first discovered in 1979, that with a delay of hours potentially mitigate or eliminate both non-radiogenic and radiogenic targeted and non-targeted damage at all levels of complex biological systems over extended periods of time that may last from a day to more than a year. These adaptive protections (APs) under genetic control may induce damage prevention (detoxification, scavenging of toxins), damage repair especially of DNA (i.e. of base changes, single-strand breaks, and double-strand breaks), and damage removal (mainly by apoptosis, cell senescence and immune responses). APs may outweigh damage caused by low doses be they from acute or protracted exposures and thus have a hormetic effect. APs do not appear to occur beyond a certain upper dose limit, which varies with tissues and cell systems and seems to be well below $\sim 500 \mathrm{mGy}$ acute exposure. An exception here is apoptosis, the incidence of which apparently increases linearly over a dose range beyond $500 \mathrm{mGy}$.

After doses below $\sim 100$ mGy there is no statistically significant increase but rather a reduction in cancer incidences in epidemiological and experimental studies. Using a model that covers both damage and protection following low-dose exposure of adult humans shows that APs preventing only about $2-3 \%$ of endogenous life-time cancer risk would fully balance a calculated induced cancer risk at about $100 \mathrm{mSv}$, in agreement with epidemiological data and concordant with a hormetic effect.

Low-dose-risk modeling must recognize signal-induced upregulation of protection leading to an approach that accommodates radiobiological information into policy and decision making and reduces unreasonable fear and unnecessary expenditure. Moreover, potential clinical applications of low-dose irradiation appear worthwhile considerations.

THE GAP JUNCTION AS A "BIOLOGICAL ROSETTA
STONE": IMPLICATIONS TO EVOLUTION, STEM CELLS,
AND HOMEOSTATIC REGULATION OF HEALTH AND DISEASE

James E. Trosko

Dept. Pediatrics/Human Development, College of Human Medicine, Michigan State University, East Lansing, Michigan 48824

The relatively new discovery of the gap junction structure, its functions and, more recently, the family of the "connexin" genes, has been basically ignored by the major biological disciplines. Yet its appearance in the early evolution of the metazoan, its fundamental functions, (e.g., to synchronize electrotonic and metabolic functions of societies of cells, and its role in regulating cell proliferation, cell differentiation, and apoptosis, via linking, in integrating the extra-cellular triggering of intra-cellular signaling, and therefore, regulating epigenetic gene expression) has been well documented by both genetic mutations of the connexin genes and by chemical modulation of gap junction function. With various genetic alteration of connexins in knock-out and transgenic mice, as well as inherited connexin mutations in various human syndromes, gap junctions and chemical modulation of gap junction function, the gap junction has been shown to be directly linked to many normal cell functions and multiple diseases, such as birth defects, reproductive, neurological disorders, immune dysfunction and cancer. Specifically, the modulation of gap junctional intercellular communication (GJIC), either by increasing or decreasing its functions by non-mutagenic chemicals or by oncogenes or tumor suppressor genes in normal or "initiated" stem cells and their progenitor cells, can have a major impact on tumor promotion or cancer chemoprevention and chemotherapy. The overview of the roles of the gap junction in the evolution of the metazoan and its potential in understanding the a "systems" view of human health and aging and the diseases of aging will be attempted.

\section{MIR-17 92 MICRORNA CLUSTER: THE MASTER REGULATOR OF TGFB SIGNALING AND CCN PROTEINS}

Michael Dews ${ }^{1}$, Jamie Fox ${ }^{1,2}$, and Andrei Thomas-Tikhonenko ${ }^{1,2}$

${ }^{1}$ Division of Cancer Pathobiology, Department of Pathology \& Laboratory Medicine, The Children's Hospital of Philadelphia Research Institute, and ${ }^{2}$ Cancer Biology Graduate Program, University of Pennsylvania School of Medicine, Philadelphia, PA 19104

Human carcinomas commonly harbor mutations in the KRAS and MYC proto-oncogenes and the TP53 tumor suppressor gene. All three genetic lesions are potentially pro-angiogenic, but in a murine colon cancer 
model, overexpression of Myc was necessary to induce vigorous vascularization. This correlated with downregulation of anti-angiogenic thrombospondin-1 and related proteins such as CCN family members, most notable CCN2 (CTGF) and CCN5 (Wisp-2). Both CCN2 and CCN5 are predicted targets for repression by the miR-17-92 microRNA cluster, which was upregulated in Myc-overexpressing cells. Indeed, miR-17-92 knockdown with antisense 2'-O-methyl oligoribonucleotides partly restored CCN2 expression and conversely transduction with a miR-1792-encoding retrovirus reduced CCN2 levels (Dews et al., 2006). Consistent with these findings, in human cancer cell lines, levels of the miR-17 92 primary transcript (MIR17HG) exhibit a very strong negative correlation with those of CCN2 and CCN5. Interestingly, CCN1 transcript also negatively correlates with MIR17HG, although it has no predicted sites for miR-17 92 family members. We hypothesize that this is because miR-17 92 targets several regulatory components in the TGF $\beta$ signaling pathway and blunts TGF $\beta$ signaling (Dews et al., 2010) which is known to enforce $\mathrm{CCN} 1$ expression. Taken together, our results suggest that miR17 92 might be a master regulator of $\mathrm{CCN}$ family members through a combination of direct and TGF $\beta$-mediated effects.

Dews, M., Fox, J. L., Hultine, S., Sundaram, P., Wang, W., Liu, Y. Y., Furth, E., Enders, G. H., El-Deiry, W. S., Schelter, J. M., Cleary, M. A., and Thomas-Tikhonenko, A. (2010). The Myc-mir-17 92 axis blunts TGF $\beta$ signaling and production of multiple TGF $\beta$-dependent antiangiogenic factors. Cancer Res 70, 8233-8246.

Dews, M., Homayouni, A., Yu, D., Murphy, D., Sevignani, C., Wentzel, E., Furth, E. E., Lee, W. M., Enders, G. H., Mendell, J. T., and ThomasTikhonenko, A. (2006). Augmentation of tumor angiogenesis by a Myc-activated microRNA cluster. Nat Genet 38, 1060-1065.

\section{CARTILAGE-SPECIFIC OVEREXPRESSION OF CCN2/CTGF PROTECTS ARTICULAR CARTILAGE FROM AGE-RELATED OSTEOARTHRITIS-LIKE CHANGES}

Shinsuke Itoh ${ }^{1,2}$, Takako Hattori ${ }^{1}$, Nao Tomita ${ }^{1,2}$, Eriko Aoyama $^{3}$, Takashi Yamashiro $^{2}$, Masaharu Takigawa ${ }^{1}$

${ }^{1}$ Department of Biochemistry and Molecular Dentistry; ${ }^{2}$ Department of Orthodontics Okayama University Graduate School of Medicine, Dentistry and Pharmaceutical Sciences and ${ }^{3}$ Biodental Research Center, Okayama University Dental School, Okayama, Japan

Objective. CCN family 2/Connective tissue growth factor (CCN2/CTGF) promotes proliferation and differentiation of chondrocytes and enhances production of extracellular matrices during endochondral ossification. To examine the role of CCN2/CTGF in maintaining the cartilage phenotype, we generated transgenic mice which overexpress CCN2/CTGF under the Col2al promoter. Since the transgenic mice showed enhanced accumulation of cartilage matrix in developing and growing stages, we investigated adult mouse articular cartilage to see whether the overexpression of CCN2/ CTGF protected knee joint cartilage from age-related degeneration.

Methods. To overexpress CCN2/CTGF in cartilage, HA-tagged ccn2/ ctg $f$ cDNA and IRES-LacZ as an expression marker were cloned under the $6 \mathrm{~kb}$ Col2al promoter-enhancer. Expression of transgene in mouse articular cartilage was detected by X-gal staining. Knee joints from 21-months old [5 TG: male 1, female 4, and 1 wild type (WT), male] and 18-months old littermates (WT: male 3, female 2) were analyzed radiographically and histologically. Proteoglycan accumulation was analyzed by toluidine blue-staining. Immunostaining was performed with anti-type I, II, and X collagens, anti-MMP13, and anti-CCN2/ CTGF antibodies. Results were analyzed statistically.

Results. Overexpression of the CCN2/CTGF gene was seen after $\mathrm{X}$-gal staining in 14-, 40-, and 60-days-old articular cartilage. Immunohistochemical analysis of CCN2/CTGF in 21 and 18 months old mice shows enhanced accumulation of CCN2/CTGF in TG articular cartilage as compared to WT, indicating that the overexpressed CCN2/CTGF had accumulated during aging of articular cartilage. Radiographic analysis of 21- and 18-month old knee joints showed that $50 \%$ (3 out of 6 ) of WT, but not TG, had narrowed joint spaces and rough cartilage surfaces indicative of osteoarthritic changes. Histological analysis showed stronger toluidine blue staining in TG than WT articular cartilage. Furthermore, immunohistochemical analysis of type X and I collagens and MMP13 showed significantly reduced staining in TG cartilage as compared to WT.

Conclusion. Continuous cartilage-specific overexpression of CCN2/ CTGF during developing and growth period caused accumulation of $\mathrm{CCN} 2 / \mathrm{CTGF}$ and reduced osteoarthritis-like degenerative changes in aged articular cartilage. Because of its stabilizing activity for articular cartilage CCN2/CTGF may be considered to be an anti-aging factor for knee joints.

\section{REGULATION OF THE SMOOTH MUSCLE CELL CYCLE BY CCN5}

Kristina C Wiesman ${ }^{1}$, Lan Wei $^{2}$, John Castellot ${ }^{1,2}$

${ }^{1}$ Program in Pharmacology and Experimental Therapeutics, Sackler School of Graduate Biomedical Sciences, Tufts University School of Medicine, Boston MA 02111. ${ }^{2}$ Program in Cell, Molecular and Developmental Biology, Sackler School of Graduate Biomedical Sciences, Tufts University School of Medicine, Boston MA 02111.

CCN5, originally identified as a growth arrest-specific gene, inhibits the motility and proliferation of rat vascular (VSMC) and human uterine smooth muscle cells (UtSMC) both in vitro and in vivo. Preliminary gene expression microarray data suggest that CCN5 increases p27 and p21 mRNA and decreases cyclin D1 mRNA expression. Forced expression of CCN5 in VSMC released from growth arrest delays cyclin D1 protein expression. Similarly, growth arrested VSMCs infected with a lentivirus expressing CCN5 have decreased cyclin D1 protein compared to growth arrested, uninfected VSMCs. Infection of exponentially growing UtSMC with lenti-CCN5 inhibits their proliferation. Interestingly, in preliminary experiments, over-expression of CCN5 in exponentially growing UtSMC does not alter cyclin D1 protein levels compared to uninfected controls. In addition, preliminary data suggests little or no change in expression of p21 or p27 in UtSMC infected with lenti-CCN5 compared to uninfected controls. However, we do observe an increase in total ERK2 protein, an increase in phosphorylation of ERK1, and an increase the phosphorylation of p70 S6kinase in CCN5 over-expressing UtSMC compared to uninfected controls. This is paradoxical, as increased activation of ERK1/ 2 and p70 S6 kinase is associated with G1 cell cycle progression and positive cell cycle regulators. These data suggest that CCN5 expression may affect proliferation of vascular and uterine smooth muscle cells by different mechanisms. Moreover, the CCN5-induced decrease in UtSMC proliferation compared to controls is accompanied by an activation of two distinct kinases that promote cell cycle progression, suggesting a possible 
dissociation of the antiproliferative activity of CCN5 and ERK-mediated signaling pathways in UtSMC. We are currently working to understand these differences in how CCN5 regulates proliferation and cell cycle control mechanisms in vascular and uterine smooth muscle cells.

\section{MULTIPLE REGULATION OF HUMAN CCN1 VIA THE 3' UTR-UNTRANSLATED REGION AND ITS BIOLOGICAL SIGNIFICANCE}

Kumi Sumiyoshi ${ }^{1.2}$, Satoshi Kubota ${ }^{1}$, Yosuke Nakagawa ${ }^{1}$, Masanao Minato $^{2}$, Toshihiro Ohgawara $^{3}$, Tsuyoshi Shimo ${ }^{3}$, Kazumi Kawata ${ }^{1}$, Takashi Nishida $^{1}$, Takashi Yamashiro ${ }^{2}$, Masaharu Takigawa ${ }^{1}$

${ }^{1}$ Department of Biochemistry and Molecular Dentistry; ${ }^{2}$ Department of Othodontics and ${ }^{3}$ Department of Oral Surgery, Okayama University Graduate School of Medicine, Dentistry and Phamceutical Sciences, Okayama, Japan

$\mathrm{CCN} 1(\mathrm{Cyr} 61)$ has been known as a mediator of widespread biological functions including cell proliferation, differentiation and adhesion. However, the regulatory mechanism of $\mathrm{CCN} 1$ gene has not been fully clarified. We have investigated the 3'-untranslated region(UTR) mediated regulatory mechanism of $\mathrm{CCN} 1$ gene and identified few segments in the $3^{\prime}$-UTR region, which were thought to be involved in the posttranscriptional regulation of the $\mathrm{CCN} 1$ gene. The first one was a secondary-structed RNA segment located in the former-half of the 3'-UTR, whereas the other was a putative target of microRNA181a (miR-181a). MicroRNAs are small non-coding RNAs that repress the translation of target genes and modulate biological process. To seek possible miRNAs targeting to the 3 '-UTR region of the CCN1 mRNA, we performed in silico assay and found miR-181a. Indeed, the overexpression of miRNA181a in chondrocytic HCS-2/8 and breast cancer MDA-231 cells led to the repression of $\mathrm{CCN} 1$ protein production from both cells. Furthermore, miR-181a overexpression resulted in the repression of aggrecan and typeIIcollagen mRNA expression in HCS-2/8 cells. These results show that miRNA181a reduces chondrocytic phenotype of HCS- $2 / 8$ cells. Therefore, miRNA may have important role in regulating the expression of the CCN1 gene and chondrocytic phenotype, both of which are related each other.

\section{RETINOIDS SUPPRESS CYSTEINE-RICH PROTEIN 61 (CCN1), A NEGATIVE REGULATOR OF COLLAGEN HOMEOSTASIS, IN SKIN EQUIVALENT CULTURES AND AGED HUMAN SKIN IN VIVO}

Taihao Quan*, Zhaoping Qin, Yuan Shao, Yiru Xu, John J. Voorhees, Gary J. Fisher*

Department of Dermatology, University of Michigan Medical School, Ann Arbor, Michigan, USA

Alterations of connective tissue collagen are prominent features of both chronologically aged and photoaged (aging due to sun exposure) human skin. These age-related abnormalities are mediated in part by CCN family member, CCN1 (cysteine-rich protein 61). CCN1 is elevated in the dermis of both chronologically aged and photoaged human skin in vivo, and promotes aberrant collagen homeostasis by down-regulating type I collagen, the major structural protein in skin, and promoting collagen degradation. Vitamin A and its metabolites have been shown to improve chronologically aged and photoaged skin by promoting deposition of new collagen and preventing its degradation. Here we investigated regulation of $\mathrm{CCN} 1$ expression by retinoids in skin equivalent cultures and chronologically aged and photoaged human skin in vivo. In skin equivalent cultures, all-trans retinoic acid (RA), the major bioactive form of vitamin $\mathrm{A}$ in skin, significantly increased type I procollagen, and reduced collagenase (matrix metalloproteinases-1, MMP-1). Addition of recombinant human $\mathrm{CCN} 1$ to skin equivalent cultures, significantly reduced type I procollagen and increased MMP-1. Importantly, all-trans retinoic acid significantly reduced $\mathrm{CCN} 1$ expression in skin equivalent cultures. Topical treatment with retinol (vitamin A, 0.4\%) for seven days significantly reduced $\mathrm{CCN} 1 \mathrm{mRNA}$ and protein expression in both chronologically aged (80+years) and photoaged human skin in vivo, compared to vehicle-treated skin. These data indicate that the mechanism by which retinoids improve aged skin, through increased collagen production, involves down-regulation $\mathrm{CCN} 1$.

\section{GSK3 $\beta$ : A NOVEL TISSUE-SPECIFIC REGULATOR of CCN2 EXPRESSION IN HUMAN GINGIVAL FIBROBLASTS}

\section{Maha Bahammam and Philip C. Trackman}

Boston University Henry M. Goldman School of Dental Medicine, Department of Periodontology and Oral Biology, Boston, MA USA

Introduction. Gingival overgrowth is a tissue-specific side-effect of certain medications including phenytoin, calcium channel blockers (e.g., nifedipine) and cyclosporine A. The incidence of overgrowth is high and leads to interference with mastication, speech, and oral hygiene and systemic complications. Cessation of drug treatment or its substitution is not always possible. Hence, development of therapies that target molecular and cellular mediators of gingival overgrowth are needed.

TGF $\beta$ promotes fibrosis, and the effects of TGF $\beta$ on gingival fibrosis are partly mediated by up-regulation of Connective Tissue Growth Factor (CTGF/CCN2) by tissue-specific signaling pathways. Hence, our laboratory is interested in identifying tissue-specific signaling pathways of $\mathrm{CTGF} / \mathrm{CCN} 2$ regulation in an effort to develop therapeutic strategies to prevent or treat gingival overgrowth

Methods. Primary human gingival fibroblasts and fetal human lung fibroblasts were cultured for comparative analysis of $\mathrm{CTGF} / \mathrm{CCN} 2$ protein expression induced by TGF $\beta 1$. The role of PI3' kinase and its downstream targets, PKC family members and GSK $3 \beta$ were analyzed using pharmacological inhibitors, siRNA mediated knockdown techniques, and expression of constitutively active forms of signaling intermediates.

Results. We first investigated a role for PI3'-kinase in mediating TGF- $\beta 1$ stimulated CTGF/CCN2 levels in primary human gingival fibroblast cultures utilizing the pharmacologic inhibitor wortmanin, and found that wortmanin blocks TGF $\beta 1$ induced CTGF/CCN2 expression. Because GSK3 $\beta$ can act downstream of PI3' kinase, we next investigated the role of GSK3 $\beta$ in mediating TGF $\beta 1$ induced $\mathrm{CTGF} / \mathrm{CCN} 2$. Findings indicate that in contrast to lung fibroblasts, inhibitory phosphorylation of GSK $3 \beta$ unexpectedly inhibits, rather than stimulates CTGF/CCN2 levels, thus identifying a potential therapeutic target. Because GSK-3 $\beta$ inhibition is a mediator of the $\mathrm{Wnt} / \beta$-catenin pathway we investigated cross- 
regulation with TGF- $\beta$ signaling, and found that GSK3 $\beta$ inhibition is independent of TGF- $\beta 1$ stimulated up-regulation of CTGF/ $\mathrm{CCN} 2$ in gingival fibroblasts. Investigations of PKC pathways indicate that they are not involved in TGF- $\beta 1$ regulation of CTGF/ $\mathrm{CCN} 2$ in human gingival fibroblasts.

Conclusion. Data identify GSK3 $\beta$ as a potential therapeutic target to treat or prevent gingival oral fibrotic diseases driven by TGF- $\beta$ and $\mathrm{CTGF} / \mathrm{CCN} 2$. These findings expand our list of potential therapeutic agents to ameliorate human gingival overgrowth that now includes statins, forskolin, and now also GSK3 $\beta$ inhibitors. Our data further demonstrate the unique regulation of $\mathrm{CTGF} / \mathrm{CCN} 2$ expression in gingival fibroblasts and elucidates molecular pathways that are likely to be important in the tissue-specificity of drug-induced gingival overgrowth.

Supported by NIH/NIDCR R01 DE11004

\section{NATURAL COMPOUNDS AS INHIBITORS OF INFLAMMATION AND ACTIVATORS OF CELL DEATH MECHANISMS}

\section{$\underline{\text { Marc Diederich }}$}

Laboratoire de Biologie Moléculaire et Cellulaire du Cancer, Hôpital Kirchberg, 9, rue Edward Steichen, L-2540 Luxembourg, Luxembourg

Programmed cell death plays a critical role in the cellular housekeeping, and aberrant cell death regulation leads to body malformations and to numerous diseases, including various autoimmune diseases, cancer, stroke, infarctations, and neurodegenerative diseases. Research in the field of cell death has evolved at a very rapid pace over the last fifty years. It is now generally accepted that programmed cell death can be subdivided into three different categories: apoptosis (programmed cell death type I), autophagy (programmed cell death type II, or lysosomal cell death), and oncosis (programmed cell death type III, also referred to as necrosis). Apoptosis, in particular, has been studied in great depth and autophagy has recently sparked an enormous research interest.

In the 1960's to early 1980 's, at the same time as renowned biologists including Richard Lockshin and John Saunders, John Kerr, Alastair Currie, and Andrew Wyllie, and Sydney Brenner, Robert Horvitz, and John Sulston initiated their investigations into cell death, research groups lead by Paul Scheuer, John Faulkner, George Pettit, and other chemists pioneered the research field of natural products, with the aim to identify potent anti-cancer compounds amongst the toxins produced by marine organisms as a chemical defence mechanism against their predators. Since then, numerous marine natural products have been identified as modulators of cell death. Here, we discuss the mechanism of action of the major marine natural products reported as inducers of apoptosis, autophagy, or oncosis.

Glucocorticoid Receptor Ligands, dissociating between Transrepression and Transactivation.

\section{Haegeman Guy}

Glucocorticoid hormones (GCs) remain the mainstay for the treatment of various inflammatory disorders, because of their great efficacy. The long-term usage of GCs is, however, overshadowed by the occurrence of debilitating side-effects, like osteoporosis, skin and muscle atrophy, diabetes and neurological disorders.

GCs exert their functions through binding to the glucocorticoid receptor (GR), a transcription factor that regulates gene transcription in a positive or negative way. Direct binding of activated GR in the promoter of target genes is believed to be the main pathway leading to metabolic gene expression (mainly hold responsible for the unwanted side-effects), whereas the interference of GR with the activity of other transcription factors, such as NF- $\mathrm{kB}$ or AP-1, greatly contributes to its desired anti-inflammatory capacities. 'Dissociated ligands' thus aim to separate GR-mediated transcriptional activation from transcriptional repression in order to achieve better side-effect profiles.

In this respect, a newly characterized, plant-derived, non-steroidal GR modulator, i.e. Compound $\mathrm{A}(\mathrm{CpdA})$ was tested both in vitro and in vivo for its dissociative effects.

Methodology. we have used CpdA in several cellular in-vitro assays as well as in in-vivo disease models to test its dissociated properties, as compared to glucocorticoids.

Results. CpdA behaves as a potent (although weaker) anti-inflammatory agent, both in-vitro as in-vivo, as compared to the synthetic glucocorticoid Dexamethasone. However, as opposed to steroidal ligands, CpdA does not give rise to the gene-activating effects in cells, nor to increased blood glucose levels or hyperinsulinemia in the tested animals. Furthermore, as opposed to glucocorticoids, CpdA does not lead to GR desensitization.

Conclusions. it is possible to fully dissociate the gene-activating effects from the inhibitory actions of GR by imposing a monomeric structure to the receptor by so-called 'specific GR modulators' (SGRMs), like CpdA. Moreover, GR desensitization can be avoided which adds to the beneficial effects for long-term treatments.

\section{"NOVEL REGULATORS OF PRO-INFLAMMATORY SIGNALING PATHWAYS"}

Prof. Paul Moynagh

Institute of Immunology, National University of Ireland Maynooth, County Kildare, Ireland

Toll-like receptors (TLRs) detect and respond to conserved microbial structures or products of microbial metabolism known as pathogenassociated molecular patterns (PAMPs). The signalling pathways triggered by TLRs share much in common with those activated by the IL-1 receptor due to the presence of a conserved intracellular Toll/IL-1 receptor (TIR) domain. Following ligand stimulation, TLRs/IL-1 receptor associate with TIR domain-containing adaptor proteins, such as MyD88, leading to the recruitment of members of the IL-1 receptor-associated kinase (IRAK) family of serine/threonine kinases (2). IRAK-1 then interacts with the downstream signalling molecule TRAF6 that in turn activates TAK1. The latter stimulates I $\mathrm{KB}$ kinases that trigger the transcription factor NF- $\mathrm{KB}$ and MKK3/6 and MKK4 that activate the MAP kinases p38 and JNK, respectively. Ubiquitination of key signaling molecules in these pathways is central to regulation of innate immune signalling. Here we focus on some recently discovered novel regulators of ubiquitination and their functional relevance to inflammation and innate immunity.

\section{MODULATION OF APOPTOSIS BY SYNTHETIC COX-2 INHIBITORS: NEW UNEXPECTED INSIGHTS FROM HEMATOLOGICAL CANCER MODELS}

Claudia Cerella ${ }^{1}$, Cyril Sobolewski ${ }^{1}$, Sebastien Chateauvieux ${ }^{1}$, Michael Schnekenburger ${ }^{1}$, Mario Dicato ${ }^{2}$, and Marc Diederich ${ }^{1 *}$

${ }^{1}$ Laboratoire de Biologie Moléculaire et Cellulaire du Cancer, Hôpital Kirchberg, Luxembourg; ${ }^{2}$ Department of Hematology-Oncology, Centre Hospitalier Luxembourg 
Cyclooxygenase-2 (COX-2) is a pro-inflammatory immediate early response gene, found stably expressed in a number of adherent cancers, where a causative role in tumor promotion as well as in determining chemotherapy failure has been suggested. Consequently, the use of preferential/selective COX-2 inhibitors in combination with traditional chemotherapeutic agents has taken into account as a strategy to potentiate COX-2 expressing cancer cells death. More recently, a stable expression of COX-2 has been found also in hematological malignancies, where similar pro-carcinogenic effects have been hypothesized.

In this study, we analyze the role of synthetic COX-2 enzyme inhibitors (nimesulide, Ns-398 and celecoxib) in survival/apoptosis on hematological cancer models. We found that COX-2 inhibitors, unexpectedly, strongly prevent apoptosis induced by a panel of chemotherapeutic agents on a selected COX-2-positive acute myeloid leukemia model. The data collected are consistent with a prevention of the apoptotic signaling at very early steps, prior to Bax/Bax activation.

Here, we provide evidence that this modulator role is due to two converging anti-apoptotic mechanisms, both depending on the exacerbation of such chemoresistance phenomena, one affecting the correct intracellular accumulation of chemotherapeutic drugs and the other hampering the activation of JNK signaling pathway. Accordingly, COX-2 inhibitors enhance drug efflux, as assessed by rhodamine 1,2,3 and doxorubicin efflux assays, and attenuate chemotherapeutic-induced DNA damage on one side. Concomitantly, they up-regulate the glutathione-Stransferase isoform GST-PI and prevent JNK signaling pathway activation, absolutely required for the cytotoxicity of the chemotherapeutic agents we tested, on the other side. Consequently, COX-2 inhibitors do not exert any anti-apoptotic potential when the cells were challenged with physiological stimuli (i.e., with TNFalpha and FAS) as well as by the stressing agent hydrogen peroxide, both neither targeted by multidrug channels nor affected by cellular GST levels.

Our findings show a novel paradoxical anti-apoptotic effect of COX-2 inhibitors on hematopoietic COX-2-positive cancer cells. This recommends cautions in the use of anti-inflammatory agents as chemoadjuvant as well as in counteracting side adverse effects of chemotherapy during the treatment of such tumors.

\section{INTERPLAY BETWEEN NON-DEATH AND DEATH TNFR IN INFLAMMATION}

Emmanuel Dejardin, Ph.D

GIGA-Research, University of Liège, Belgium, Unit of Molecular Immunology and Signal Transduction

Inflammation is an important biological response for healing. However, acute and chronic inflammations are sometimes a threat for the human body, which ultimately can cause inflammatory disorders and even cancer development. The transcription factor $\mathrm{NF}-\kappa \mathrm{B}$ is a ubiquitous factor that controls the transcription of many genes, whose aberrant expression is a key event in the development of the pathology. We will present new mechanistic insights on the mechanisms involved in activation of NF-kB. More importantly, we will depict how NF-kB-related inflammation might be linked to TNFR1-induced cell death and cancer progression.

\section{MECHANISMS OF INSULIN-LIKE GROWTH FACTOR BINDING PROTEIN-3 CELLULAR UPTAKE AND NUCLEAR DELIVERY}

Lucia Micutkova ${ }^{1}$, Martin Hermann ${ }^{2}$, Martin Offterdinger ${ }^{3}$, Michael W. Hess ${ }^{4}$, Andrea Matscheski ${ }^{1}$, Haymo Pircher ${ }^{1}$, Christoph Mück ${ }^{1}$, Hannes-Leonhard Ebner ${ }^{4}$, Andreas Laich ${ }^{1}$, Elisa Ferrando-May ${ }^{5}$, Werner Zwerschke ${ }^{1}$, Lukas A. Huber ${ }^{6}$, Pidder Jansen-Dürr ${ }^{1}$

${ }^{1}$ Institute for Biomedical Aging Research, Innsbruck, Austria; ${ }^{2} \mathrm{KMT}$ Laboratory, Innsbruck Medical University, Innsbruck, Austria; ${ }^{3}$ Biocenter, Division of Neurobiochemistry/Biooptics, Innsbruck Medical University, Innsbruck, Austria; ${ }^{4}$ Division of Histology and Embryology, Innsbruck Medical University, Innsbruck, Austria; ${ }^{5}$ University of Konstanz Bioimaging Center, Konstanz, Germany; ${ }^{6}$ Biocenter, Division of Cell Biology, Innsbruck Medical University, Innsbruck, Austria

Extracellular signalling molecules are involved in the regulation of many different cellular processes and diseased conditions. Insulin-like growth factor binding proteins (IGFBPs) and IGFBP-related proteins, also referred as CCN (Cyr61, CTGF and Nov) family, are two related extracellular protein families, which share a similar amino terminal domain (Kim et al., PNAS, 1997). Members of both protein families were linked with processes like cell survival, apoptosis, cell migration, carcinogenesis and aging. The main function of IGFBPs, as extracellular proteins, is to control the bioavailability of IGFs and thus control cell growth, apoptosis and differentiation (for review see Firth and Baxter, Endocr Rev, 2002). CCN proteins are also capable of IGF binding, however with considerable lower affinity, and most of the CCN actions seem to be IGF-independent (for review see Holbourn et al., Trend Biochem Sci, 2008). Interestingly, several recent studies suggest, that some of the IGFBPs, including IGFBP-3, -5 and -6 , can also modulate cellular activities in an IGF-independent manner. This activity seems to involve the uptake of IGFBPs from the extracellular space and their translocation into the nucleus. In this study, we focused on one of the members of IGFBPs family, IGFBP-3. It has been reported, that IGFBP-3 mutants, that do not bind IGFs, can still stimulate apoptosis in cancer cells (Santer et al., Cancer Res, 2006; Hong et al., JBS, 2002). Furthermore, it has been shown that extracellular IGFBP-3 can be internalized by endocytosis and translocated into the nucleus (Schedlich et al., JBC, 2000). Nevertheless, the precise mechanisms underlying IGFBP-3 cellular uptake and intracellular distribution are unknown. In this study we have investigated the internalization of IGFBP-3 by live cell imaging, backed up by electron microscopical and biochemical studies. We demonstrate that, IGFBP-3 is internalized in a dynamin-dependent process involving both clathrin-mediated and caveolar endocytosis, and confirm the presence of IGFBP-3 in the nucleus of living cells.

This work was supported by PROTEOMAGE and the Austrian Science Funds through SFB 021 and FSP 093.

\section{MOLECULAR ELUCIDATION OF WISP1 FUNCTIONS IN HMSCS AND CHONDROCYTES}

Katrin Schlegelmilch ${ }^{1}$, Alexander Keller ${ }^{2}$, Viola Zehe ${ }^{1}$, Norbert Schütze ${ }^{1}$

${ }^{1}$ University of Würzburg, Orthopedic Institute, Orthopedic Center for Musculoskeletal Research, Würzburg, Germany; ${ }^{2}$ University of Würzburg, Department of Bioinformatics, Würzburg, Germany 
WISP1 (wnt1 inducible signaling pathway protein 1, CCN4) is a matrix associated secreted signal molecule that belongs to the $\mathrm{CCN}$ family. Members are characterised by an almost identical modular structure. Further, they have essential functions in development, angiogenesis, proliferation and other fundamental cell processes. Molecular data on WISP1 functions in the musculoskeletal system are limited, but the known association with the wnt/ß-catenin pathway indicates an important role during skeletal development. In this study we investigate global gene expression patterns and self-regulated expression of WISP proteins in the musculoskeletal system.

Human bone marrow-derived mesenchymal stem cells (hMSCs) were isolated from the femoral head obtained from patients undergoing total hip arthroplasty. Recombinant (r) WISP-Fc-tagged proteins were expressed in baculovirus infected insect cells (SF-21) and purified with protein Gsepharose. Western blotting and silver staining were applied to verify protein size and purity. Cells obtained from the chondrocyte cell line Tc28a2 were treated with $500 \mathrm{ng}$ rWISP1 isoforms T1 or T2, or rWISP2 to investigate selfregulatory processes. The amount of endogenous WISP1 in hMSCs and Tc28a2 target cells was reduced by application of shRNA. Different shRNA- and control-constructs (scrambled, GFP, vector without insert) were transfected into human embryonic kidney cells (HEK 293-T) via lipofectamine. Virus supernatant was used to infect target cells. After 4 8 days total RNA was isolated from WISP1 knock down cells as well as corresponding control cells and analysed by RT-PCR and microarray experiments (Affymetrix HG-U133 Plus 2.0, Tc28a2 cells: $n=5$ and hMSCs: $n=3$ ).

In the baculovirus expression system, four $150 \mathrm{~cm}^{2}$ cell culture flasks yielded 200-400 $\mu \mathrm{g}$ protein. Silver staining and western blotting revealed a protein purity of $>95 \%$ and a single band of the expected size. RT-PCR analyses detected a remarkable decrease of WISP1 (both isoforms) expression during the knock down experiments. In contrast to this, RT-PCR signal intensity remained unchanged in control cells (scrambled, GFP, vector without insert). A self-regulatory expression (positive feedback) was observed with RT-PCR after treatment with rWISP1-T1 and -T2 in knock down Tc28a2 cells. WISP2 expression was not detectable in native Tc28a2 cells, but after treatment with WISP2. Microarray analyses revealed a large number of differentially expressed genes in both cell systems. For these, we identified differences in gene clusters (e.g. interferone inducible genes in hMSCs) as well as pathway representations (e.g. MAPK pathway in Tc28a2).

Different sets of upregulated genes are involved in immune response processes. Overrepresented gene regulation in pathways often referred to the mediation of cell division, cell cycle and apoptosis. Both observations implicate an important role of WISP1 during skeletal development.

\section{MYOCARDIAL CONNECTIVE TISSUE GROWTH FACTOR (CCN2/CTGF) ATTENUATES LEFT VENTRICULAR REMODELING AFTER MYOCARDIAL INFARCTION AND PREVENTS ISCHEMIC HEART FAILURE}

Jørgen Gravning ${ }^{1,2}$, Stein Ørn ${ }^{3,4}$, Thor Edvardsen ${ }^{1,2,5}$, Vladimir N. Martinov $^{1,2}$, Cord Manhenke ${ }^{3,4}$, Kenneth Dickstein ${ }^{4}$, Håvard

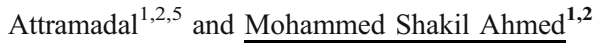

${ }^{1}$ Institute for Surgical Research, Oslo University Hospital, Rikshospitalet, Norway; ${ }^{2}$ Center for Heart failure research, University of Oslo, Oslo, Norway; ${ }^{3}$ Division of Cardiology, Stavanger University Hospital, Norway; ${ }^{4}$ Institute of Internal Medicine, University of Bergen, Norway; ${ }^{5}$ Department of Cardiology, Oslo University Hospital, Rikshospitalet, Norway
Purpose. Myocardial CCN2/CTGF - connective tissue growth factor is induced in experimental models of heart failure as well as in human heart failure. However, its pathophysiological role in the development of ischemic heart failure remains unresolved.

Methods. Transgenic mice with cardiac-restricted overexpression of CTGF (Tg-CTGF) were compared with nontransgenic littermate control mice (NLC). Myocardial infarction (MI) was induced by ligation of the left coronary artery in Tg-CTGF $(n=22)$ and NLC mice $(n=21)$ and left ventricular (LV)remodeling and cardiac function was assessed after 4 weeks. Area at risk was estimated in a separate group of animals after perfusion with Evans blue dye, and was similar among Tg-CTGF and NLC mice. In addition, serum levels of CTGF (s-CTGF) were measured in 42 patients admitted to hospital for STelevation myocardial infarction (MI), 2 days, 1 week, 2 months and 1 year after percutaneous coronary intervention (PCI). Cardiac magnetic resonance imaging was performed at the same time points to determine infarct size and LV ejection fraction (EF).

Results. During the 4 weeks follow-up, there was significantly better survival in Tg-CTGF mice as compared to NLC mice; $63.6 \%$ vs. $38.1 \%, p<0.05$. In vivo pressure-volume analysis after 4 weeks displayed preserved cardiac performance in Tg-CTGF mice, as measured by dp/dt max, LV end-diastolic and end-systolic pressure as well as cardiac output, and end-point analysis after excision of the hearts revealed attenuation of cardiac hypertrophy and pulmonary congestion in Tg-CTGF mice vs NLC mice (Heart weight/body weight ratio; $5.3 \pm 0.2 \mathrm{mg} / \mathrm{g}, n=14$ vs $8.0 \pm 0.9 \mathrm{mg} / \mathrm{g}, n=9, p<0.05$ ). Also, markers of myocardial remodeling, i.e. myocardial BNP and betamyosin heavy chain mRNA levels, measured by real time qPCR analysis, were significantly less up-regulated in Tg-CTGF than in NLC hearts. Interestingly, in patients in which s-CTGF levels increased from day 2 after PCI until 2 months after PCI $(n=21)$, infarct healing was significantly improved and LV remodeling attenuated one year after the ischemic event. Consistently, EF was also significantly higher in these patients after one year, as compared to patients with unaltered or decreased s-CTGF levels $(n=21)$.

Conclusion. CTGF prevents development of experimental ischemic heart failure in mice, and increase in s-CTGF levels in patients after MI is associated with attenuated LV remodeling and improved cardiac function. These results may indicate cardioprotective effects of CTGF in ischemic heart failure.

\section{WISP-1/CCN4 REGULATES OSTEOGENESIS BY ENHANCING BMP-2 ACTIVITY}

Mitsuaki Ono ${ }^{1,2}$, Colette A. Inkson ${ }^{1}$, Tina M. Kilts ${ }^{1}$, Yasutaka Oida ${ }^{2}$, Wataru Sonoyama $^{2}$, Takuo Kuboki ${ }^{2}$ and Marian F. Young ${ }^{1}$

${ }^{1}$ Molecular Biology of Bones and Teeth Section, Craniofacial and Skeletal Diseases Branch, NIDCR, NIH; ${ }^{2}$ Department of Oral Rehabilitation and Regenerative Medicine, Okayama University Graduate School of Medicine, Dentistry and Pharmaceutical Sciences

Wnt-induced Secreted Protein-1 (WISP-1/CCN4) is a member of the $\mathrm{CCN}$ family that is highly expressed in skeletal tissue and in osteoprogenitor cells induced to differentiate in vitro. To determine the function of WISP-1 during osteogeneis, osteogenic bone marrow stromal cells (BMSCs) were transduced with WISP-1 adenovirus (adWISP-1) in the presence or absence of BMP-2 adenovirus (adBMP-2). WISP-1 over-expression enhanced the ability of BMP-2 
to direct BMSCs towards osteogenic differentiation and appeared to work by stimulating Smad 1/5/8 phosphorylation and activation. The ability of WISP-1 to enhance BMP-2 activity was also shown in vivo using and ectopic osteogenesis assay with BMCS transduced with WISP-1, BMP-2 or both. When BMSCs were infected with lentivirus containing human WISP-1 shRNA they formed less bone in vivo and were less responsive to BMP-2 confirming that WISP-1 and BMP-2 have a functional interaction. Immunoprecipitation (IP) and western analysis showed that WISP-1 bound directly to BMP-2 and showed that WISP-1 increased BMP-2-binding to hBMSCs in a dosedependent fashion. To understand how WISP-1 enhanced BMP-2 signaling the influence of WISP-1 on integrin expression was analyzed. WISP-1 induced the mRNA and protein levels of alpha5 integrin and, further, was found to bind to it. Antibody blocking experiments showed that the BMP-2 binding to BMSC that was enhanced by WISP-1 was completely neutralized by treatment with anti-integrin alpha5beta1 antibody. Pilot studies and using transgenic mice that over-expressed human WISP-1 in pre-osteoblasts had increased BMD, trabecular thickness and BV/TV compared to wildtype controls supporting observations using human osteoprogenitors that WISP-1 has a positive influence on osteogenesis in vivo. In conclusion, these studies show, for the first time, that WISP-1 has a positive influence on bone cell differentiation and function and may work by enhancing the effects of BMP-2 to increase osteogenesis through a mechanism potentially involving binding to integrin alpha5beta1. Supported by the IRP-NIDCR 1 Z01 DE000379-21.

\section{CCN2 EXERTS DIRECT CYTOPROTECTIVE ACTIONS ON CARDIAC MYOCYTES BY ACTIVATION OF THE PI3- KINASE/AKT/GSK-3 $\beta$ SIGNALING CASCADE}

Ingvild Tronstad $\mathrm{Moe}^{1}$, Tuyet Anh Pham ${ }^{1}$, M. Shakil Ahmed ${ }^{1}$ and Håvard Attramadal ${ }^{1,2}$

${ }^{1}$ Institute for Surgical Research and ${ }^{2}$ Dept. of Cardiology, Rikshospitalet Medical Center and University of Oslo, Oslo, Norway

Background. The matricellular protein $\mathrm{CCN} 2$ is repressed in healthy hearts, but its expression in the heart is induced in heart failure. The purpose of this study was to investigate whether cardiac myocytes are direct targets of CCN2 and to resolve to what extent the cardioprotective effects of $\mathrm{CCN} 2$ could be due to activation of cytoprotective signaling.

Methods. Adult mouse cardiac myocytes were isolated from C57/BL6 mice by retrograde perfusion with collagenase. We investigated the effects of recombinant human CCN2 (rec hCCN2) on survival of cardiac myocytes under hypoxia, oxidative stress, and prolonged culturing. Cardiac myocytes were preincubated in the absence or presence of $200 \mathrm{nmol} / \mathrm{L}$ rec hCCN2 for 20 hours, and subsequently exposed to $15 \mathrm{umol} / \mathrm{L}$ Doxorubicin for additional 20 hours, or exposed to hypoxia $(1 \% \mathrm{O} 2)$ for 4 hours. Adenylate kinase (AK) activity and release of lactate dehydrogenase $(\mathrm{LDH})$ into cell culture medium were quantified. Protein/phosphoprotein levels were analyzed by Western blot of extracts from cardiac myocytes after stimulation in the presence or absence of rec hCCN2.

Results. Cardiac myocytes incubated with rec hCCN2 tolerated hypoxia ( $\mathrm{LDH}$ released into the medium in Control vs CCN2 treated cells; $1,40 \pm$ 0,08 vs $0,96 \pm 0,07 P=0.0019, n=6$; relative units) and oxidative stress (LDH released into the medium of Doxorubicin vs Doxorubicin $+\mathrm{CCN} 2$ treated cells; $2.5 \pm 0.06$ vs $1.9 \pm 0.05 P<0.0001 \quad n=6$; relative units) better than controls, and had higher long-term survival (48 hours) (LDH released into the medium in Control vs CCN2 treated cells; $1.8 \pm 0.07$ vs $1.3 \pm 0.03, P<0.0001, n=6$; relative units). Western blot analysis showed that rec hCCN2 phosphorylates Akt (Ser 473) and GSK-3 $\beta$ Ser 9) in concentration dependent manner. The CCN2-stimulated phosphorylation of Akt was sensitive to PI3-kinase inhibition (LY294002), and the phosphorylation of GSK-3 $\beta$ was sensitive to both PI3-kinase inhibition and Akt inhibition (API-2), demonstrating activation of a PI3-kinase/ Akt/GSK-3 $\beta$-signaling pathway. PI3-kinase inhibition (LY294002) abolished the cytoprotective effect of rec hCCN2.

Conclusions. Cardiac myocytes are direct target cells of CCN2 and that CCN2 activates PI3 kinase-dependent Akt/GSK-3ßphosphorylation with ensuing inhibition of GSK-3 $\beta$ activities leading to robust cytoprotection towards hypoxia and oxidative stress.

\section{CCN2/CTGF BINDS TO FIBROBLAST GROWTH FACTOR RECEPTOR 2 AND MODULATES ITS SIGNALING}

Eriko Aoyama $^{1}$, Satoshi Kubota ${ }^{2}$, Masaharu Takigawa ${ }^{1,2}$

${ }^{1}$ Biodental Research Center, Dental School, Okayama University, Okayama, Japan and ${ }^{2}$ Department of Biochemistry and Molecular Dentistry, Okayama University Graduated School of Medicine, Dentistry and Pharmaceutical Science, Okayama, Japan

CCN2/CTGF has a critical role in mesenchymal tissues such as cartilage and bone. CCN2/CTGF deficient mice show that it is essential in normal development and metabolism of bone. It is also known that CCN2/CTGF binds to several cytokines and receptors and regulates their signaling. We carried out protein array to find unidentified partners of CCN2/CTGF and some candidates were discovered. Among them, we selected fibroblast growth factor receptors (FGFR) 2 and 3 for further investigation, because some point mutations in FGFR caused some hereditary bone disorders (e.g., Apert syndrome, Crouzon disease et al.). We used solid-phase binding assay to ascertain the binding of CCN2/CTGF to FGFR2 and FGFR3. The results indicated that both of them could bind to CCN2/ CTGF, but the binding of FGFR2 to CCN2/CTGF was more distinct than that of FGFR3. Therefore, we focused on the binding of CCN2/ CTGF to FGFR2 and performed surface plasmon resonance (SPR) to calculate dissociation constant $(\mathrm{Kd})$ between them. The level of $\mathrm{Kd}$ between them $(7.71 \mathrm{nM})$ was very close to those between FGFR2 and its known ligands. Next, we found TSP1 module of CCN2/CTGF was concerned with the binding by means of solid-phase binding assay using anti-module antibodies. Interestingly, the bindings of FGFR2 and known ligands, FGF2 and FGF4, were enhanced in the presence of $\mathrm{CCN} 2 / \mathrm{CTGF}$, which means that $\mathrm{CCN} 2 / \mathrm{CTGF}$ supported their binding as a cofactor. In the osteoblastic cell line MC3T3-E1, CCN2/ CTGF had a collaborative effect on phosphorylation of ERK by FGF2 stimulation. As a conclusion, we are going to present a result indicating the biological significance of the binding of CCN2/CTGF to FGFR2 in bone metabolism.

\section{CCN2/CTGF-A NOVEL STIMULATOR OF CARDIAC STEM CELL PROLIFERATION VIA ACT- DEPENDENT REGULATION OF CYCLIN D2}

$\underline{\text { Vladimir N Martinov A }}{ }^{1}$, M. Shakil Ahmed ${ }^{1}$, Einar O Vik-Mo ${ }^{1}$, David Kunke $^{2}$, Magnar Bjørås ${ }^{2}$, Håvard Attramada ${ }^{1}$

${ }^{1}$ Institute for Surgical Research and ${ }^{2}$ Institute of Microbiology Oslo Univ Hosp, Oslo, Norway 
Stem cell therapy is a novel tentative treatment option for myocardial infarction. Challenges are more effective means of proliferation and survival of cardiac stem cells, as well as development of procedures for transplantation and engraftment of cardiac stem cells in the heart. In this study we investigate the function of the matricellular protein CCN2 on stem cell proliferation and survival.

Methods. Cardiac stem cells were isolated from mouse myocardial tissue and maintained in IMDM/CEM media to stimulate formation of cardiospheres as reported (Messina et al.CircRes. 2004;95:911-921). Immunocytochemical analysis confirmed the phenotypic characteristic of cardiac stem cells positive for Sca-1, C-kit, Gata4, Nkx-2.5 and Isl-1. Cell proliferation was analyzed by EdU Flow Cytometry. Cardiac progenitor cells exposed to $\mathrm{H}_{2} \mathrm{O}_{2}$ damage $(100 \mu \mathrm{mol} / \mathrm{L})$ were compared in the presence or absence of CCN2 $(300 \mathrm{nmol} / \mathrm{L})$. Recombinant human CCN2 was purified from the cell culture media of HEK293 cells and purified by sequential affinity (heparin-Sepharose) and ion-exchange (SSepharose) chromatography to more than $95 \%$ purity.

Results. Rec-hCCN2 stimulated cardiosphere formation and growth. CCN2 stimulated proliferation of cardiac stem cell by enhancing progression through S and G2 phase of the cell cycle. CCN2-stimulated cell cycle progression was concentration dependent and correlated with AKT/GSK-3 $\beta$ phosphorylation (EC50 250 nmol/L). Cell cycle progression was shown to be dependent on AKT/GSK-3 $\beta$ signaling (abrogated by PI3 kinase inhibitor LY294002 and AKT-inhibitor API-2) and was associated with robust upregulation of cyclin D2 mRNA expression. Survival of cardiac progenitor cells was significantly increased in the presence of CCN2 as assayed by activity of cellular reductases (MTT Survival Assay).

Conclusion. In the current study we demonstrate that CCN2 stimulate proliferation and survival of cardiac stem cells by activation of AKT/GSK-
$3 \beta$ signaling and upregulation of cyclin-D2. CCN2 may become an important factor for amplification and survival of cardiac stem cells in stem cell therapy.

[poster or oral requested]

\section{INHIBITION OF FAT CELL DIFFERENTIATION (FCD) BY CCN-2 IS DEPENDENT ON TRANSFORMING GROWTH FACTOR-B (TGF-B) PATHWAY SIGNALING.}

William C. Song ${ }^{1}$, Susan V. McLennan ${ }^{1,2}$, Paul F. Williams ${ }^{1,2}$, $\underline{\text { Stephen M. Twigg }}$

${ }^{1}$ Discipline of Medicine, University of Sydney and ${ }^{2}$ Department of Endocrinology, Royal Prince Alfred Hospital, Sydney. NSW, Australia

FCD affects insulin sensitivity and lipid storage. In vitro, our lab has demonstrated that the protein CCN-2 inhibits FCD in NIH3T3L1 cells and in rodent adipocyte primary cultures. While the cell mechanism is unknown, in other systems CCN-2 action can be TGF- $\beta$ pathway dependent. The aim of this study was to investigate in FCD the dependence of the CCN-2 effect on TGF- $\beta$ and TGF- $\beta$ downstream pathway signaling.

NIH3T3-L1 cells were differentiated at d0 using standard methods with IBMX/Dex/Insulin. FCD at d10 was measured by increased gene markers and lipid accumulation. Cells were treated at $\mathrm{d} 0$ with single dose active rhTGF- $\beta 1(2 \mathrm{ng} / \mathrm{mL})$, rh CCN-2 (500 ng/mL) and/or TGF- $\beta$ type 1 receptor blocker (SB431542,5 $\mu \mathrm{M}$ ), up to $48 \mathrm{~h}$. FCD key transcription factors: CEBPs and PPAR- $\gamma$ were also determined.

\begin{tabular}{|c|c|c|c|c|c|c|c|}
\hline & \multicolumn{7}{|c|}{ Day10 Post differentiation } \\
\hline & $\begin{array}{l}\text { Differentiated } \\
\text { alone }\end{array}$ & $\begin{array}{l}\text { TGF- } \beta \text { R1 blocker } \\
\text { alone }\end{array}$ & $\begin{array}{l}\text { DMSO } \\
\text { vehicle }\end{array}$ & $\begin{array}{l}+\mathrm{rhCCN}- \\
2\end{array}$ & $\begin{array}{l}\text { +rhCCN-2+TGF- } \beta \mathrm{R} 1 \\
\text { blocker }\end{array}$ & $\begin{array}{l}+ \text { rhTGF- } \\
\beta 1\end{array}$ & $\begin{array}{l}+ \text { rhTGF- } \beta 1+\text { TGF- } \beta 1 \\
\text { blocker }\end{array}$ \\
\hline $\begin{array}{l}\text { Adiponectin } \\
\left(\mathrm{x} 10^{4}\right)\end{array}$ & $4.34 \pm 1.75^{*}$ & $4.04 \pm 1.20 *$ & $\begin{array}{l}2.90 \pm \\
0.76^{*}\end{array}$ & $\begin{array}{c}1.63 \pm \\
0.85^{\#}\end{array}$ & $4.96 \pm 2.54^{*}, \#$ & $\begin{array}{r}1.10 \pm \\
0.51\end{array}$ & $3.87 \pm 1.83^{* !}$ \\
\hline Resistin (x10) & $4.73 \pm 1.38^{*}$ & $4.16 \pm 1.08^{*}$ & $\begin{array}{c}3.02 \pm \\
0.53 *\end{array}$ & $\begin{array}{c}2.08 \pm \\
0.39^{\#}\end{array}$ & $3.15 \pm 0.45^{*}, \# \#$ & $\begin{array}{c}2.04 \pm \\
0.88^{\#}\end{array}$ & $3.99 \pm 0.39^{*}, !$ \\
\hline ORO staining & 2.82 & 2.79 & 2.80 & 2.61 & 2.80 & 2.58 & 2.85 \\
\hline
\end{tabular}

Data are fold change \pm SD compared with Non-differentiated cells. ${ }^{*} p<0.05$ vs. Non-differentiated; $\# p<0.05$ vs. Differentiated alone; $\# \# p<0.05$ vs rh CCN-2; $p<0.05$ vs. rhTGF- $\beta 1$, all by ANOVA.

$\mathrm{CCN}-2$ inhibition of FCD markers adiponectin and resistin was prevented by TGF- $\beta$ receptor blockade (Table) as was lipid accumulation. Similar prevention was found using pan-specific neutralising antiTGF- $\beta$ antibody (not shown). Both CCN-2 and TGF- $\beta$ treatment rapidly phosphorylated SMAD-3 signaling in early stages of FCD. Furthermore, in an early time course of $2 \mathrm{~h}$, single doses of rhTGF- $\beta 1$ or rhCCN-2 inhibited by $\sim 30 \%$ (each $p<0.05$ ) the induction of C/EBP- $\beta$ and $-\delta$ mRNA and protein levels otherwise seen during FCD.

This work shows that CCN-2 effects on FCD are both TGF- $\beta$ and TGF- $\beta$ pathway dependent and are related to early effects on C/EBPs. Further studies will determine how SMADs are utilised by $\mathrm{CCN}-2$ in this process.
Supported by NHMRC of Australia

\section{INHIBITORY EFFECT OF CCN3 ON ARTERIAL NEOINTIMAL HYPERPLASIA THROUGH MODULATION OF SMOOTH MUSCLE CELL GROWTH AND MIGRATION}

Koutaro Yokote, Tatsushi Shimoyama, Shu-ichi Hiraoka, Takahiko Tokuyama, Masaki Fujimoto, Yuya Tsurutani, Bernard Perbal, Haruhiko Koseki, Minoru Takemoto

Department of Clinical Cell Biology and Medicine, Chiba University Graduate School of Medicine; RIKEN Research Center for Allergy and Immunology; Laboratoire d'Oncologie Virale et Mole'culaire 
CCN3 belongs to the $\mathrm{CCN}$ family, which constitutes multifunctional secreted proteins that act as matrix cellular regulators. We investigated the pathophysiological roles of $\mathrm{CCN} 3$ in the vessels. We examined the effects of $\mathrm{CCN} 3$ on the proliferation and migration of rat vascular smooth muscle cells (VSMC). CCN3 knockout mice were created, and vascular phenotypes and neointimal hyperplasia induced by photochemically induced thrombosis were investigated. CCN3 suppressed the VSMC proliferation induced by fetal bovine serum. The neutralizing antibody for transforming growth factor- $\beta$ did not affect the growth inhibitory effect of $\mathrm{CCN} 3$. Moreover, $\mathrm{CCN} 3$ enhanced the mRNA expression of cyclin-dependent kinase inhibitors, $\mathrm{p} 21$ and $\mathrm{p} 15 . \gamma$ secretase inhibitor, an inhibitor of Notch signaling, partially inhibited the enhanced expression of $\mathrm{p} 21$ induced by $\mathrm{CCN} 3$. CCN3 also inhibited the VSMC migration. Finally, the histopathologic evaluation of the arteries 21 days after the endothelial injury revealed a 6-fold enhancement of neointimal thickening in the null mice compared with the wild-type mice. In conclusion, $\mathrm{CCN} 3$ suppresses neointimal thickening through the inhibition of VSMC migration and proliferation. Our findings indicate the involvement of CCN3 in vascular homeostasis, especially on injury, and the potential usefulness of this molecule in the modulation of atherosclerotic vascular disease.

\section{CCN3 PROMOTES NEURITE OUTGROWTH}

\section{Wun-Chey Sin}

Department of Cellular and Physiological Sciences, Life Sciences Institute, The University of British Columbia, Vancouver, BC, Canada.

Little is known about the role of CCN3 in the central nervous system although it is widely expressed in the brain. An earlier study showed that its RNA expression is developmentally regulated in postnatal rats, increasing from birth and peaking between P30 and P60. Similar results were obtained from in situ hybridization on adult mouse brain carried out by the Allen Brain Atlas Project. The correlation of CCN3 levels with early postnatal brain development suggests that $\mathrm{CCN} 3$ is likely to have a role in neuronal morphogenesis.

The cellular localization of $\mathrm{CCN} 3$ in the mouse brain was further examined with anti-CCN3 antibodies. High $\mathrm{CCN} 3$ protein levels were detected in the cell bodies and the proximal processes of pyramidal neurons in layer $2 / 3$ and in layer 5 of the cerebral cortex. The protein was also detected in the CA1 but not in the CA3 region of the hippocampus. The result is in agreement with the finding of GENSAT (Gene expression Nervous System Atlas), confirming that CCN3 has a neuronal distribution in the mouse brain. In cultured mouse neocortical neurons of 8 DIV (days in vitro), CCN3 protein was expressed at a higher level in MAP2positive dendrites than in Tau-positive axonal processes. It is especially enriched in growth cones at the tip of neurites, suggesting CCN3 might actively participate in regulating neurite outgrowth. Western analysis on conditioned medium from primary culture revealed significant level of $\mathrm{CCN} 3$ secreted by the cortical neurons from 10 DIV onwards, indicating that $\mathrm{CCN} 3$ expression is associated with neuronal maturation. At $18 \mathrm{DIV}$, $\mathrm{CCN} 3$ protein was detected as a single $48 \mathrm{kDa}$ protein in the conditioned medium while a smaller $35 \mathrm{kDa}$ protein was present exclusively in the cell pellet.

We next determine whether introduction of $\mathrm{CCN} 3$ to mouse cortical neurons for 48 hours at 0 DIV will alter their growth characteristics. Exogenous application of $\mathrm{CCN} 3$ protein increased both the cumulative length and number of neurites. Overexpression of an intracellular form of CCN3 in the cortical neurons also promotes branching. Taken together, our data suggests that $\mathrm{CCN} 3$ facilitates neuronal morphogenesis possibly by interaction of extracellular CCN3 with cell surface receptors and through intracellular signaling initiated by the non-secreted isoform.

\section{CCN2-EXPRESSING PERICYTES ARE REQUIRED FOR BLEOMYCIN-INDUCED SKIN SCLERODERMA}

Shangxi Liu ${ }^{1}, \mathrm{Xu}$ Shi-wen ${ }^{2}$, David J. Abraham ${ }^{2}$ and Andrew Leask ${ }^{1}$

${ }^{1}$ Division of Oral Biology and Department of Physiology and Pharmacology, Schulich School of Medicine and Dentistry, University of Western Ontario, Dental Sciences Bldg., London, ON, Canada, N6A 5 C1 and ${ }^{2}$ Centre for Rheumatology, University College London (Royal Free Campus), Rowland Hill St., London, UK, NW3 2PF

The pro-adhesive matricellular protein connective tissue growth factor (CTGF/CCN2) is a marker of fibrotic cells and is significantly overexpressed in scleroderma. Our objective was to determine whether CCN2 was required for bleomycin-induced skin scleroderma and to investigate the origin of fibrotic fibroblasts within fibrotic skin lesions. To perform this analysis, we subjected adult mice bearing a smooth muscle cell/fibroblast-specific deletion of $\mathrm{CCN} 2$ to the bleomycin-induced model of skin scleroderma. In wild-type mice, immunohistochemical analysis with anti-CCN2 and anti-NG2 antibodies showed that the myofibroblasts induced in response to bleomycin expressed both $\mathrm{CCN} 2$ and the pericyte-specific marker NG2 $(N=6, p<0.01)$. In response to bleomycin, wild-type, but not CCN2-deficient, mice showed increased skin thickness, as visualized by hematoxylin and eosin staining, and collagen production as visualized by trichrome staining and hydroxyproline/proline analysis $(N=6, p<0.05)$. Moreover, in response to bleomycin, wild-type mice possessed, but CCN2-deficient mice lacked, abundant NG2/ $\alpha$-SMAexpressing myofibroblasts within fibrotic lesions $(N=6, p<0.05)$. Collectively, these results indicate that $\mathrm{CCN} 2$-expressing pericytes are essential for bleomycin-induced skin scleroderma. These data indicate that therapeutic strategies blocking $\mathrm{CCN} 2$ /pericyte differentiation in vivo may be of benefit in combating the skin fibrosis in scleroderma.

\section{CCN2/CTGF INTERACTS WITH MATRILIN-3 AND ENHANCES ASSEMBLY OF A CARTILAGE MATRIX PROTEIN AND FILAMENTOUS NETWORK}

Takako Hattori ${ }^{1}$, Daisuke Araki ${ }^{1}$, Mitsuhiro Hoshijima ${ }^{1}$, Eriko Aoyama ${ }^{2}$, Choichiro Shinmura ${ }^{1}$, Christiane Otten $^{3}$, Raimund Wagener ${ }^{3}$, Masaharu Takigawa ${ }^{1}$

${ }^{1}$ Department of Biochemistry and Molecular Dentistry, Okayama University of Graduate School of Medicine, Dentistry and Pharmaceutical Sciences; ${ }^{2}$ Biodentental Research Center, Okayama University Dental School, Okayama Japan and ${ }^{3}$ University of Cologne, Cologne, Germany

To identify proteins that interact with connective tissue growth factor (CCN2/CTGF) and modulate its action, we carried out a yeast-two hybrid screen using ccn2 and a cDNA library from the chondrocytic cell line HCS-2/8. As a major CCN2-interacting protein we found matrilin-3, a non-collagenous extracellular matrix protein, which associates with collagen and plays a role in collagen 
fibril assembly. Here we demonstrate that $\mathrm{CCN} 2$ binds to matrilin-3 in a dose-dependent manner and specify the interaction sites in both molecules. CCN2 colocalized with matrilin-3 and collagen type IX in the pericellular and intercellular matrix of mouse primary chondrocytes. CCN2 enhanced adhesion of the human chondrocytic cell line HCS-2/8 to matrilin-3. An antibody against matrilin-3 which inhibited adhesion of HCS-2/8 cells to matrilin-3 did, however, not inhibited CCN2-enhanced adhesion to matrilin-3, indicating a separate CCN2binding site on the cell surface. Furthermore, CCN2 seemd to modulate formation of fibril networks containing collagen IX collagen in the intercellular space. CCN2-deficient chondrocytes showed less pericellular and intercellular collagen IX than wild type chondrocytes. Addition of recombinant $\mathrm{CCN} 2$ to the culture medium enhanced intercellular collagen IX-positive network formation, co-distributing with matrilin-3. In a direct binding assay, CCN2 did not significantly affect matrilin-3 binding to collagen IX. The results of a competition assay, however, showed CCN2 enhanced the affinity of matrilin-3 binding to collagen IX. These results suggest that $\mathrm{CCN} 2$ supports multiple specific interactions of extracellular cartilage proteins, in particular matrilin-3, and their assembly on the chondrocyte surface, as well as cell-matrix interactions.

\section{THE KALLIKREIN-RELATED PEPTIDASE 12 HYDROLYZES MATRICELLULAR PROTEINS OF THE CCN FAMILY AND MODIFIES INTERACTIONS OF CCN1 AND CCN5 WITH GROWTH FACTORS}

Audrey Guillon-Munos ${ }^{1}$, Katerina Oikonomopoulou ${ }^{2}$, Noémie Michel ${ }^{1}$, Chistopher R. Smith ${ }^{2}$, Agnès Petit-Courty ${ }^{1}$, Sylvie Canepa ${ }^{3}$, Pascale Reverdiau ${ }^{1}$, Nathalie Heuzé-Vourc' ${ }^{1}{ }^{1}$, Eleftherios P. Diamandis ${ }^{2}$, Yves Courty ${ }^{1}$

From INSERM U618 -Université François Rabelais ${ }^{1}$, Faculté de Médecine, 2 bis bd Tonnellé, 37032 Tours, France, Department of Pathology and Laboratory Medicine ${ }^{2}$, Mount Sinai Hospital, Toronto, Ontario, M5G1X5, Canada, and the Biacore laboratory, UMR INRACNRS-Université de Tours-Haras Nationaux ${ }^{3}, 37380$ Nouzilly France

Kallikrein-related peptidases (KLKs) are an emerging group of secreted serine proteases involved in several important physiological and pathological processes. In this study, we used a degradomic approach to identify potential substrates of KLK12. MDA-MB-231 cells were treated either with KLK12 or vehicle control in serum-free medium and the proteome of the overlying media was analyzed by mass spectrometry. The matricellular protein $\mathrm{CCN} 1$ was amongst the proteins released by the KLK12-treated cells, suggesting that KLK12 might be responsible for the shedding of this protein from the cell surface. Fragmentation of CCN1 by KLK12 was further confirmed in vitro and the main cleavage site was localized in the hinge region between the first and second half of the recombinant protein. Additional in vitro studies revealed that KLK12 can target all six members of the $\mathrm{CCN}$ family at different proteolytic sites, ranging from one for CCN5 to 9 for CCN4. Solid-phase binding assay and surface plasmon resonance analysis showed that fragmentation of $\mathrm{CCN} 1$ or CCN5 by KLK12 prevents $\mathrm{VEGF}_{165}$ binding, while it also triggers the release of intact VEGF and BMP-2 from the CCN complexes. The KLK12-mediated release of TGF- $\beta 1$ and FGF-2, either as intact or truncated forms, were found to be concentration-dependent. These findings suggest that KLK12 may indirectly regulate the bioavailability and activity of several growth factors through processing of their $\mathrm{CCN}$ binding partners. Decrease in the growth factor functionality can potentially influence several biological processes including angiogenesis, bone and bone marrow homeostasis and tumourigenesis.

\section{SCREENING OF CCN2-BINDING PEPTIDES AND ITS SCIENTIFIC UTILITY}

Satoshi Kubota ${ }^{1}$, Eriko Aoyama ${ }^{2}$, Harumi Kawaki ${ }^{1}$, Hirohisa Mitsunaga ${ }^{1}$, Naoya Fujita ${ }^{3}$, Kenta Nakai ${ }^{3}$ and Masaharu Takigawa ${ }^{1,2}$

${ }^{1}$ Department of Biochemistry and Molecular Dentistry, Okayama University Graduate School of Medicine, Dentistry and Pharmaceutical Sciences, Okayama, Japan; ${ }^{2}$ Biodental Research Center, Okayama University Dental School, Okayama, Japan; ${ }^{3}$ Human Genome Center, Institute of Medical Science, University of Tokyo, Tokyo, Japan

$\mathrm{CCN} 2$ and other $\mathrm{CCN}$ family protein members are composed of conserved 3 or 4 modules that are highly interactive to a variety of biomolecules. Such interaction with other moieties is considered to be a major background supporting the multiple functionalities of $\mathrm{CCN}$ family proteins. Therefore, seeking for functional counterparts is of critical importance to uncover the working mechanism of CCN family proteins. In order to obtain comprehensive information concerning the binding counterparts of $\mathrm{CCN} 2$, we screened a random dodecapeptide library displayed on a filamentous bacteriophage library with each independent modular protein of $\mathrm{CCN} 2$ as a probe to find out $\mathrm{CCN} 2$ binding peptide sequences.

Recombinant human CCN2 single modular proteins were produced by trasnsformed gram-positive bacteria, Brevibacillus choshinensis, through natural secretion, which ruled out the possibility of endotoxin contamination. However, dimodular, trimodular and full-length CCN2 proteins could not be obtained by this novel method. After purification, the single modules were coated onto a plastic well cluster, and selection and cloning of bacteriophages were performed by 3 rounds of biopanning. Consequently, we obtained $9,11,4$ and 9 different dodecapeptide sequences that bound to IGFBP, VWC, TSP1 and CT modules of human CCN2, respectively. These peptide sequences were utilized to develop our research in 2 different manners: On the one hand, a common peptide motif was extracted from all of the dodecapeptides binding to the same module in silico, and was utilized to design aptamers that would bind to $\mathrm{CCN} 2$ and alter its function. On the other hand, each dodecapeptide sequence was used as a key to search a human protein database to specify candidates of CCN2 binding counterparts.

Until now, we have designed several $\mathrm{CCN} 2$ aptamers and evaluated their functionality, based on the former approach. At least, one such aptamer was found to modify CCN2 function. As a result of the latter approach, we are identifying novel CCN2-binding proteins that play significant roles in bone biology. Recent advance concerning this issue will be presented on site.

\section{FUNCTION OF CONNECTIVE TISSUE GROWTH FACTOR IN HUMAN PULMONARY MICROVASCULAR ENDOTHELIAL AND HUMAN MESENCHYMAL STEM CELLS}

Roderich Laug ${ }^{1}$, Susanne Jatzke ${ }^{1}$, Viola Monz ${ }^{1}$, Steffen Kunzmann ${ }^{2}$, Norbert Schütze ${ }^{1}$

${ }^{1}$ Orthopedic Center for Musculoskeletal Research, University of Wuerzburg, Germany; ${ }^{2}$ University Children's Hospital, Wuerzburg, Germany 
Human connective tissue growth factor (CTGF/CCN2) belongs to the $\mathrm{CCN}$ family. These signalling and regulatory molecules are involved in many different cellular processes such as angiogenesis, migration and differentiation. The $\mathrm{CCN}$ family is characterised by the presence of an N-terminal signal, followed by four modular structural domains (IGFBP,- von Willebrand,- thrombospondin,- and the carboxyterminal domain) and 38 cysteines residues (except CCN5) that are mostly conserved within the members of the family. Aim of this study is to identify the influence of recombinant CTGF (rCTGF) on human mesenchymal stem cells (hMSCs) and human pulmonary microvascular endothelial cells (HPMECs) and to analyse the differential gene expression between CTGF knock down and control cells by microarray analysis.

The open reading frame of CTGF fused to the Fc-domain of human $\operatorname{IgG}$ was cloned into the baculovirus expression vector pBacPAK8. SF21 insect cells were transfected with CTGF and recombinant expressed protein was purified by affinity chromatography on G-Sepharose columns. Different shRNAs, to target CTGF RNA, were transfected into human embryonic kidney cells (HEK293T). Knock down of CTGF in the target cells was achieved by lentiviral infection with the virus supernatant. hMSCs and HPMECs were cultured in serum reduced media and treated with recombinant protein for $24 \mathrm{~h}$. RNA was isolated $24 \mathrm{~h}$ after treatment. Differential gene expression was analysed by microarray analysis of Affymetrix (Human Genome U133 plus 2.0).

The yield of recombinant protein was about $200-350 \mu \mathrm{g} / \mathrm{ml}$ from four $150 \mathrm{~cm}^{2}$ flasks. Western blotting and silver staining showed a single band of rCTGF at the expected size with high purity. Downregulation of endogenous protein expression in hMSCs and HPMECs could clearly be shown by RT-PCR and western blotting compared to control cells, while RT-PCR signals in cells that received scrambled and GFP oligos remained unchanged. The microarray analysis of CTGF treated versus untreated knock down cells couldn't show any differences in gene expression. However the untreated knock down cells compared with cells that received the scrambled oligo detected numerous and reproducible regulations in gene expression $(n=3)$. Scores of regulated genes were mainly found for the MAPK,- TGFß,- Jak-STAT- and VEGF-signaling pathway.

Relating to the detected gene expression in the microarray results previously published studies showed that CTGF is connected via different pathways in lung development and lung disease. In addition the HPMEC-array and the MSC-array of this study provide the possibility to further elucidate the important role of CTGF in both target cells.

\section{CCN3 AUGMENTS IMATINIB INDUCED GROWTH INHIBITION IN CHRONIC MYELOID LEUKAEMIA}

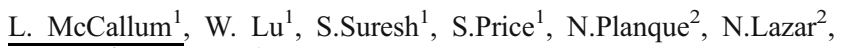
B.Perbal $^{3}$, AE.Irvine ${ }^{1}$.

${ }^{1}$ Centre for Cancer Research and Cell Biology, Queen's University Belfast, Belfast, UK.; ${ }^{2}$ Laboratoire D'Oncologie Virale et Moleculaire, Universite Paris, Paris, France and ${ }^{3}$ L'Oreal R\&D, New Jersey, USA

Chronic Myeloid Leukaemia (CML) is characterized by expression of the constitutively active BCR-ABL tyrosine kinase. Previously, we identified down-regulation of the negative growth regulator, $\mathrm{CCN} 3$, as a result of BCR-ABL kinase activity. Reduced CCN3 expression is a prominent feature in both primary human CML cells and cell lines.
We now show that $\mathrm{CCN} 3$ is growth inhibitory factor and expression enhances imatinib induced growth inhibition.

K562 cells were stably transfected with a construct containing CCN3 (pCMV82-23 (2.25 x10 $10^{6}$ copies per $50 \mathrm{ng}$ of cDNA)) and growth characteristics and activation of signaling pathways were compared to cells transfected with empty vector (control (CCN3 levels undetected by RQ-PCR). pCMV82-23 cells showed reduced colony formation capacity $(p=0.003)$ and reduced cell growth over a period of five days $(p=0.005)$. CCN3 expression caused a significant reduction in phosphorylation of ERK $(p=0.002)$, AKT $(p=0.017)$ and STAT5 $(p=0.005)$, while total protein levels were unaffected. Flow cytometry showed sustained $\mathrm{CCN} 3$ expression resulted in an accumulation of cells within the subG $\mathrm{G}_{0}$ stage of the cell cycle $(11.4 \% \pm$ $3(p=0.040))$.

To determine if $\mathrm{CCN} 3$ could influence sensitivity to imatinib, pCMV82-23 cells and control cells were treated with imatinib (5uM) for $48 \mathrm{~h}$. CCN3 expression showed a significant increase in the magnitude of imatinib induced growth inhibition $(63.3 \% \pm 10.5$ ( $p=$ $0.043)$ ) compared to control cells $(19.6 \% \pm 2.5)$ and was reflected by increased accumulation of cells in the $\mathrm{subG}_{0}$ area of the cell cycle, $34.6 \% \pm 5$ for pCMV82-23 cells compared to control cells $(21.7 \% \pm$ $8(p=0.006))$. Similarly, K562 cells treated with imatinib (5uM) in combination with recombinant $\mathrm{CCN} 3(10 \mathrm{nM})$ for $48 \mathrm{~h}$ showed enhanced growth inhibition $(71.8 \% \pm 7.9)$ compared to cells treated with imatinib alone $(81.1 \% \pm 9.2(p=0.008))$.

Expression of CCN3 in CML cells restores growth control and re-establishes induction of apoptosis. $\mathrm{CCN} 3$ expression or the addition of recombinant protein provide additional benefit for imatinib induced growth inhibition, presenting a novel avenue for therapeutic intervention.

\section{DEREGULATED MIRNA EXPRESSION CONTRIBUTES TO CCN3 DOWNREGULATION IN CHRONIC MYELOID LEUKAEMIA}

Sukanya Suresh, Lynn McCallum, Susan Price, Alexandra E. Irvine

Department of Haematology, Centre for Cancer Research and Cell Biology, Queen's University Belfast, United Kingdom

Chronic Myeloid Leukaemia (CML) is characterized by the presence of Bcr-Abl fusion gene and the constitutive expression of the Bcr-Abl tyrosine kinase oncoprotein. $\mathrm{CCN} 3$ is a negative growth regulator in CML and is downregulated by Bcr-Abl activity. MicroRNAs (miRNAs) are small noncoding RNAs that negatively regulate protein expression and have key roles in cellular regulation. In CML, Bcr-Abl causes upregulation of miRNAs favouring disease progression. CCN3 downregulation in CML could be the result of Bcr-Abl upregulation of specific miRNAs targeting the CCN3 transcript resulting in translational repression of $\mathrm{CCN} 3$ protein. This study identifies Bcr-Abl associated miRNAs targeting CCN3 in the K562 CML cell line.

Global miRNA profiling was established using TaqMan ${ }^{\circledR}$ Low Density Human MicroRNA Arrays Set v2.0 microfluidic cards (TLDA). TLDA facilitates rapid real time PCR quantitation of 667 human miRNAs in a 384 well format. These arrays specifically detect physiologically active mature miRNAs using Taqman based chemistry. To identify Bcr-Abl dependent miRNA expression, K562 cells were transfected with anti-Bcr-Abl siRNA $(0.5 \mu \mathrm{g})$ using Amaxa nucleofection technology. Bcr-Abl was reduced by 3.8 fold at $24 \mathrm{~h}$ 
and 3.3 fold at $72 \mathrm{~h}$ following siRNA treatment. A corresponding 3.4 fold increase in CCN3 was observed at $24 \mathrm{~h}(p<0.0005)$ and levels were maintained at $72 \mathrm{~h}$ (3.7 fold, $p<0.001)$. Total RNA $(1 \mu \mathrm{g})$ from K562 cells and from anti-Bcr-Abl siRNA treated cells at $24 \mathrm{~h}$ and $72 \mathrm{~h}$ was reverse transcribed using Megaplex ${ }^{\mathrm{TM}}$ RT stem loop primers and assayed with TLDA cards on a $7900 \mathrm{HT}$ fast real time PCR using ABI sequence detection software v2.3. The results were analyzed by SDS Relative Quantification (RQ) Manager using $22_{\mathrm{T}}^{-\Delta \Delta \mathrm{C}}$ method. The miRNAs that could target CCN3 were predicted using miRGEN target interface that uses four different miRNA target prediction algorithms (DIANA, PicTar, TargetScan, Miranda).

Of the 293 Bcr-Abl associated miRNAs (112 upregulated and 181 downregulated), 14 were found to target the CCN3 3' UTR, 4 of which (mir-130b, mir-130a, mir-148a, mir-425-5p) showed greater than 1.5 -fold reduction at $24 \mathrm{hrs}(n=3)$. Mir-130b showed 3.4 fold reduction $(p<0.05)$ at $24 \mathrm{~h}$, and 3.1 fold reduction at $72 \mathrm{~h}(p<0.05)$ and was selected for further studies. The human acute myeloid leukaemic cell line, HL60, is Bcr-Abl negative and expresses CCN3. HL60 cells were transfected with the pre-miRNA precursor of mir$130 \mathrm{~b}(30 \mathrm{~nm})$ and scrambled miRNA and CCN3 mRNA levels were determined. Mir-130b transfection into HL60 cells showed decreasing levels of CCN3 transcript, 1.8 fold reduction at $24 \mathrm{~h}(p<0.01)$ and 2.02 fold reduction $(p<0.05)$ at $48 \mathrm{~h}(n=3)$.

Bcr-Abl downregulation of CCN3 in CML allows leukaemic cells to evade normal growth regulation. This study shows that Bcr-Abl associated miRNA(s) including mir-130b could contribute to the repression of CCN3 in CML. Further validation of mir-130b and other miRNAs in the study may provide us with better understanding of CML and the rationale to exploit miRNAs as therapeutic strategies.

\section{INVASIVE PHENOTYPE OF CCN6 KNOCKDOWN BREAST CELLS IS MEDIATED THROUGH P38 KINASE ACTIVATION}

\section{Anupama Pal, Ph. D. and Celina G. Kleer, M.D.}

Department of Pathology and Comprehensive Cancer Center, University of Michigan Medical School, Ann Arbor, Michigan 48109, USA

CCN6 is a secretory, cysteine-rich matricellular regulatory protein which is reported to be reduced or lost in invasive carcinomas of the breast with lymph node metastasis and in inflammatory breast cancer. Previous studies from our laboratory have established the growth inhibitory and tumor suppressive functions of CCN6 in breast cancer. However, it is not known how reduction of CCN6 leads to tumor formation and invasion in the mammary glands. We hypothesized that CCN6 knockdown (CCN6 KD) in human mammary epithelial cells (HME) may disrupt normal acinar growth and promote invasion through alteration of cytoskeletal proteins. We used the 3 dimensional culture system in matrigel, a physiologically relevant ex vivo model. HME cells with lentivirus mediated stable knockdown of CCN6 were cultured on growth factor reduced matrigel for 15 days under standard culture conditions. While control HME cells formed growth arrested, polarized acini with basement membrane deposition, CCN6 KD cells showed an extremely branching and invasive phenotype with loss of Ecadherin and alpha 6 integrin. Mechanistically, CCN6 KD induced the phosphorylation of P38 kinase in cells grown on matrigel. Treatment with $500 \mathrm{ng} / \mathrm{ml}$ recombinant human CCN6 protein or the specific P38 kinase inhibitor SB203850 rescued the invasive phenotype to single, round organized structures and restored Ecadherin and alpha 6 integrin protein expression and localization. To further understand how CCN6 regulates P38 and invasion, we analyzed transcript levels of TGF beta and BMP family members. Quantitative RT-PCR showed a 21-, 20-, and 5-fold increase in the transcript levels of BMP4, and its regulators BMPER and BAMBI, respectively. We found strong induction of P38 kinase phosphorylation in response to BMP4 in CCN6 KD cells compared to controls. Of note, treatment with BMP4 function inhibiting antibody reverted $\mathrm{P} 38$ activation and invasion of CCN6 KD HME cells. Our data show that the invasive phenotype of CCN6 KD cells is mediated through BMP4 upregulation and enhanced BMP4-dependent activation of P38 kinase.

\section{RENIN-ANGIOTENSIN SYSTEM (RAS) AND CTGF: NEW ANTI-FIBROTIC THERAPEUTIC STRATEGIES ASSOCIATED WITH DUCHENNE MUSCULAR DYSTROPHY}

\section{E. Brandan, M. G. Morales and C. Cabello-Verrugio}

Laboratory of Cell Differentiation and Pathology, Department of Cell and Molecular Biology, CARE, Catholic University of Chile, Santiago, Chile. ebrandan@bio.puc.cl

Duchenne muscular dystrophy (DMD) is characterized by necrosis, degeneration and regeneration of muscle fibers that finally produces the decline in muscle function and the appearance of fibrosis. This last process can be accelerated through the exercise in the $m d x$ mice, one of the animal models of DMD. Among the factors involved in skeletal muscle fibrosis are transforming growth factor type beta (TGF- $\beta$ ), connective tissue growth factor (CTGF) and the renin-angiotensin system (RAS), whose main fibrotic exponent is angiotensin-II (AngII). Levels of CTGF are enhanced in $m d x$ skeletal muscle and genetic evidence strongly suggests that CTGF expression is directly involved in the skeletal muscle degree of fibrosis.

Objective. To evaluate the direct involvement of RAS as a therapeutic target to reduce the fibrosis associated with DMD.

Results and Discussion. Myoblasts and fibroblasts obtained from skeletal muscle increased the amount of CTGF and fibrotic markers in response to Ang-II. This induction requires AT-1 receptors since $\boldsymbol{A}$ ng-II Receptor type 1 Blockers (ARB) inhibit the fibrotic effect mediated by Ang-II. Interestingly, in vitro studies suggest that ARB decrease CTGF dependent fibrosis. In vivo experiments showed that tibialis anterior skeletal muscle from normal mice infected with an adenovirus containing mouse CTGF sequence (Adv-mCTGF) resulted in an increase of fibrosis determined by the amount of fibronectin, collagen type III and CTGF. However this increase in fibrosis was significantly diminished when the Adv-mCTGF and ARB were administered together. Sedentary and exercised $m d x$ mice, which have increased CTGF levels, developed more fibrosis than corresponding control mice, evaluated through the increased levels of collagen type III and fibronectin. The treatment of $m d x$ mice with ARB, decreased levels of CTGF and the fibrotic markers, and improved muscle function assessed by challenge of speed tests and electrophysiological assays in isolated skeletal muscle.

Conclusion. The results suggest that RAS and CTGF are potential targets for developing therapeutic anti-fibrotic strategies that could decrease muscle dysfunction associated with DMD.

FONDECYT-11080212, FONDAP-1398001, Conicyt-PFB12/2007, MDA 89419. 
CYR61 PROMOTES INTRATIBIAL TUMOR GROWTH AND LUNG METASTASIS IN A MOUSE EXPERIMENTAL OSTEOSARCOMA MODEL

Adam Sabile ${ }^{1}$, Matthias Arlt ${ }^{1}$, Roman Muff $^{1}$, Beata Bode ${ }^{2}$, Bettina Langsam ${ }^{1}$, Josefine Bertz ${ }^{1}$, Thorsten Jentzsch ${ }^{1}$, Gabor Puskas ${ }^{1}$, Walter Born ${ }^{1}$, and Bruno Fuchs ${ }^{1}$

${ }^{1}$ Laboratory for Orthopedic Research, Department of Orthopedics, Balgrist University Hospital, Zurich, Switzerland

${ }^{2}$ Institute of Surgical Pathology, University Hospital, Zurich, Switzerland

Osteosarcoma is the most frequent primary malignant bone tumor in children and adolescents with a high propensity for metastasis to the lung. Metastasis is the main cause of death of patients with metastatic Osteosarcoma, who have a mean 5-year survival rate of less than $20 \%$. Thus, novel drugs that target the complex metastatic process are urgently needed. Cyr61, a member of the CCN family of growth factors, has biological roles in angiogenesis, wound healing, and bone development, but most importantly also in tumor biology However, its biological relevance in osteosarcoma tumorigenesis and metastasis has so far not been investigated.

In the present study, immunohistochemical analysis of an Osteosarcoma tissue microarray, consisting of specimens collected form ninety patients, demonstrated abundant expression of Cyr61 in more than $89 \%$ of the tumor specimens. Cyr61 immunostaining of tumor resections correlated with metastasis and poor survival. Moreover, stable overexpression of Cyr61 in the low metastatic human SaOS-2 osteosarcoma cell line increased migration and the invasion rate in-vitro, suggesting increased in vivo metastatic potential. This was confirmed in an intratibial Osteosarcoma mouse model, reflecting the human disease with lung metastases. Overexpression of Cyr61 in SaOS-2 cells accelerated intratibial primary tumor growth and enhanced metastasis to the lung, which, as a consequence significantly decreased the survival rate of the mice. Cyr61 signaling in SaOS-2 cells through the PI3-kinase/Akt/GSK3ßpathway resulted in accumulation of the cell cycle inhibitor $\mathrm{p} 21^{\mathrm{Cip} 1 / \mathrm{WAF} 1}$ in the cytoplasm, suggesting reduced cell cycle inhibitory activity in the nucleus. In conclusion, Cyr61 is a potential novel tissue marker for poor prognosis in Osteosarcoma and appears to interfere with cell cycle control in tumor cells.

\section{CCN2/CCN3 INTERACTIONS AND ACTIVITY IN SKIN FIBROBLAST RESPONSE TO GADOLINIUM: POSSIBLE ROLE IN NEPHROGENIC SYSTEMIC FIBROSIS}

Bruce L. Riser ${ }^{1,2}$, James Varani ${ }^{3}$, and JoAnn Rambow ${ }^{1}$

${ }^{1}$ Rosalind Franklin University, Dept. of Physiology and Biophysics, North Chicago, Il; ${ }^{2}$ Baxter Healthcare Corporation, Renal Division, McGaw Park, Il; ${ }^{3}$ University of Michigan, Dept. of Pathology, Ann Arbor, MI.

Nephrogenic systemic fibrosis (NSF) is a systemic fibrotic disorder whose etiology has been linked to individuals with renal failure and exposure to one or more of the gadolinium-based contrast agents (GBCAs) during magnetic resonance imaging (MRI) procedures. Numerous, "plump" fibroblast-like cells are commonly seen in NSF skin lesions, suggesting that the disease is more of a fibroplastic disease than a true fibrosis. In our recent studies it has been shown that Omniscan and other clinically-used GBCAs directly stimulate proliferation of human dermal fibroblasts. Further, Omniscan-induced fibroblast growth is reduced in the presence of an antibody to the platelet-derived growth factor (PDGF) receptor. Concomitantly, GBCA exposure up-regulates matrix metalloproteinase-1 (MMP-1) and tissue inhibitor of metalloproteinases-1 (TIMP-1) without stimulating type I procollagen production, thus suggesting effects on collagen turnover rather than on collagen synthesis per se. Here we demonstrate that skin fibroblasts exposed to Omniscan elaborate CCN2 (CTGF), a peptide which is considered to be "down-stream" of transforming growth factor- $\beta$ (TGF$\beta$ ) in relation to collagen synthesis and "down-stream" of PDGF in regard to cell growth. This upregulation of CCN2 occurred in the absence of increased TGF- $\beta$. Further, Omniscan-stimulated fibroblasts also were shown to produce $\mathrm{CCN} 3$ (Nov), which we have recently reported to be an endogenous $\mathrm{CCN} 2$ inhibitor. Last, exogenous CCN3 treatment was shown to inhibit Omniscan-induced fibroblast proliferation, as well as the increase in MMP-1/TIMP-1 induced by the GBCA. Together, these data suggest that $\mathrm{CCN} 2 / \mathrm{CCN} 3$ are part of the internal signaling pathway regulating fibroblast responses to the MRI contrast agent and therefore likely to be important in both the development of, and resistance to, the proliferative/fibrotic process.

[oral requested]

DIABETES IS A PROGRESSION FACTOR FOR HEPATIC FIBROSIS IN A HIGH FAT FED MOUSE OBESITY MODEL OF NON-ALCOHOLIC STEATOHEPATITIS: HEPATIC CCN-2 AS A POTENTIAL MEDIATOR

Lisa Lo ${ }^{1,5}$, Susan V. McLennan ${ }^{1,2,5}$, Paul F. Williams ${ }^{1,2,5}$, James Bonner ${ }^{2}$, David R. Brigstock $^{6}$, Geoffrey W. McCaughan ${ }^{3,4,5}$, Mark D. Gorrel1 ${ }^{4,5}$, Dennis K. Yue ${ }^{1,2,5}$, Stephen M. Twigg ${ }^{1,2,5}$

Discipline of Medicine and the Bosch Institute ${ }^{1}$; Department of Endocrinology, Royal Prince Alfred Hospital ${ }^{2}$; AW Morrow Gastroenterology and Liver Centre, Royal Prince Alfred Hospital ${ }^{3}$; Liver Immunobiology, Centenary Institute ${ }^{4}$; Sydney Medical School, The University of Sydney ${ }^{5}$, NSW 2006, Australia; Center for Clinical and Translational Research, Research Institute at Nationwide Children's Hospital, Columbus, OH, USA ${ }^{6}$

Aims/hypothesis. While type 2 diabetes is an independent risk factor for worsening of human non-alcoholic steatohepatitis (NASH) in clinical studies, whether diabetes exacerbates NASH has not been systematically reported in any model. The study aim was to determine if diabetes causes NASH progression in a mouse model of diet induced obesity.

Methods. C57BL/6 mice were fed a high fat diet (HFD: $45 \% \mathrm{kCal}$ fat) or standard chow (CHOW: $12 \% \mathrm{kCal}$ fat) for 20 weeks and some animals $(\mathrm{HFD}+\mathrm{DM}$ or $\mathrm{CHOW}+\mathrm{DM})$ were also rendered diabetic by low dose streptozotocin for the final 5 weeks, to model type 2 diabetes. Serum assays included circulating insulin, triglyceride, ALT and AST, glucose and ultrasensitive CRP and results of insulin tolerance tests. Intrahepatic lipid, triglyceride, macrophage infiltration and fibrosis were determined. Fibrosis markers collagen-I, collagen-III, CCN-2/CTGF, TIMP-1 and FAP were assessed by qPCR and CTGF and collagen-I by immunostaining. Results. HFD mice were obese, insulin resistant and hyperinsulinaemic, with NASH features of elevated intrahepatic lipid and macrophages, but without fibrosis. In contrast, the HFD+DM mice exhibited fibrosis in addition to these NASH features. By ANOVA, Sirius red staining at perisinusoidal, portal tract and central vein sites, collagen-I, collagen-III, FAP and TIMP-1 transcripts and collagen-I and CCN-2 
protein were each significantly increased in HFD+DM, compared with CHOW alone. In a further experiment, insulin treatment protected against fibrosis and CRP increases in HFD+DM, showing that diabetes, not streptozotocin, causes the fibrosis..

Conclusion. This novel model indicates that diet-induced NASH fibrosis is exacerbated by diabetes and attenuated by insulin therapy. Subsequent studies will utilise $\mathrm{CCN}-2$ blocking methods that target activated hepatic stellate cells to determine if $\mathrm{CCN}-2$ mediates the hepatic fibrosis in this model. Supported by NHMRC project grant of Australia.

[oral or poster requested]

\section{FIBROBLASTS DERIVED FROM DIABETIC HEART ARE SUPERSENSITIVE TO CCN-2/CONNECTIVE TISSUE GROWTH FACTOR (CTGF)}

Xiao-Yu Wang ${ }^{1}$, Susan V. McLennan ${ }^{1,2}$, Terri J. Allen ${ }^{3}$, Christopher. Semsarian $^{4,5}$, Stephen M. Twigg ${ }^{1,2}$

Discipline of Medicine, The University of Sydney ${ }^{1}$, Department of Endocrinology ${ }^{2}$, Royal Prince Alfred Hospital, Sydney; Baker Heart and Diabetes Research Institute, Melbourne ${ }^{3}$; Agnes Ginges Centre for Molecular Cardiology, Centenary Institute ${ }^{4}$ and Department of Cardiology, Royal Prince Alfred Hospital ${ }^{5}$, Sydney, AUSTRALIA

Diabetic cardiomyopathy is characterised by interstitial fibrosis, cardiomyocyte hypertrophy and apoptosis. $\mathrm{CCN}-2$ is implicated in the fibrosis possibly through effects on cardiac fibroblasts. This study examined $\mathrm{CCN}-2$ expression in cultured cardiac fibroblasts derived from both diabetic and non-diabetic rat ventricular tissue, and effects of $\mathrm{CCN}-2$ treatment on fibrosis induction on these cells. CCN-2 cell surface receptor, Tyrosine kinase A (TrkA) and transforming growth factor-beta (TGF- $\beta$ ) signaling pathways were further investigated for $\mathrm{CCN}-2$ function on the fibrotic markers. Gene expression was measured by real-time RT-PCR for extracellular matrix (ECM) markers and their protease inhibitors. The CCN-2 and ECM proteins collagen in ventricular tissue were detected by immunohistochemistry. In passage one primary cultures of cardiac fibroblasts, CCN-2, ECM genes collagen-III (Col III), fibronectin (FN), and tissue inhibitors of matrix metalloproteinases (TIMP-1 and -2) and plasminogen activator inhibitor-1 (PAI-1) mRNA were all significantly increased in diabetic (up to 3 fold) compared with control derived heart fibroblasts. Elevated protein levels of CCN-2, Collagen-I (Col I), collagen-III and fibronectin were also observed in the diabetic heart ventricular tissue. A similar pattern was seen for passage two cells by rhCCN-2 treatment: CCN-2 induced ECM markers by $2-6$ fold in diabetic cells, which was much greater than the 1.5-2 fold induction seen in control cells. In the normal cardiac fibroblasts, $\mathrm{CCN}-2$ induced gene expression of collagen-I, collagen III, fibronectin and TIMP-1 through TrkA and TGF- $\beta$ but not on PAI-1; while in diabetic cardiac fibroblasts, $\mathrm{CCN}-2$ through TrkA and TGF- $\beta$ pathways regulates collagen III, fibronectin and TIMP-1, but not collagen I and PAI-1. In conclusion, the study shows that cardiac fibroblasts from diabetic animals have upregulated $\mathrm{CCN}-2$ and are supersensitive to $\mathrm{CCN}-2$. TrkA and TGF- $\beta$ pathways may play important roles in $\mathrm{CCN}-2$ regulation in cardiac fibroblasts.

Supported by a NHMRC Australia project grant.

\section{EXPRESSION AND FUNCTIONAL ROLE OF CCN3/NOV DURING ARTICULAR CARTILAGE DEVELOPMENT}

Danilo Janune ${ }^{1,2}$, Satoshi Kubota ${ }^{1}$, Takashi Nishida ${ }^{1}$, Noureddine Lazar ${ }^{3}$, Bernard Perbal $^{3}$, Seiji Iida ${ }^{2}$ and Masaharu Takigawa ${ }^{1}$

${ }^{1}$ Department of Oral Biochemistry and Molecular Dentistry; ${ }^{2}$ Department of Oral and Maxillofacial Reconstructive Surgery, Okayama University Graduate School of Medicine, Dentistry and Pharmaceutical Sciences, Okayama City, Okayama Prefecture, Japan and ${ }^{3}$ Laboratoire d'Oncologie Virale et Moléculaire, UFR de Biochimie, Université Paris 7, Paris, France

Cartilage has a pivotal role in the musculoskeletal system. It can be classified, based on the fate of its cells, in transient or permanent cartilage. The most typical transient cartilage is growth plate cartilage, which leads to genesis and growth of long bones like femur and ribs. A typical permanent cartilage is the articular cartilage, which does not enter the endochondral ossification pathway and remains at the joint surface of long bones. The cell signaling mechanism and molecules responsible for determining the fate of each cartilage cell type, preventing the articular cartilage cells from entering the ossification pathway, maintaining its phenotype and, thus, keeping the joint functionality is still unclear. It is known that $\mathrm{CCN}$ family members are implicated in key steps of the cartilage metabolism. CCN2/CTGF, especially, has an important role in phenotype maintenance and in differentiation orchestration of both growth plate and articular cartilage cells. On the other hand, $\mathrm{CCN} 3 / \mathrm{NOV}$ effect on articular cartilage is unknown. $\mathrm{CCN} 3 / \mathrm{NOV}$, unlike $\mathrm{CCN} 2 / \mathrm{CTGF}$, inhibits proliferation of many cell types, including costal chondrocytes. Since cartilage cells that undergo endochondral ossification are more mitotically active than the articular cartilage cells, $\mathrm{CCN} 3 / \mathrm{NOV}$ might play a pivotal role in the determination of the fate of chondrocytes. To shed light on the actual $\mathrm{CCN} 3 / \mathrm{NOV}$ role on articular chondrocyte, we isolated chondrocytes from the femoral epiphysis of 5 days old rat and, after confirming the articular chondrocyte phenotype maintenance through immunocytochemistry for aggrecan, a chondrocyte marker, and tenascin $\mathrm{C}$, a protein expressed by articular chondrocytes, but not by growth plate chondrocyte, we evaluated the effects of exogenous human recombinant $\mathrm{CCN} 3 / \mathrm{NOV}(\mathrm{hrCCN} 3)$ on proteoglycan and DNA syntheses by radioactive metabolic labeling. Our results showed that $96.92 \%$ of the cells were positive for aggrecan and $97.32 \%$ for tenascin $\mathrm{C}$ and that hrCCN3 dose dependently increases proteoglycan synthesis and diminishes DNA synthesis at $25 \mathrm{ng} / \mathrm{ml}$. Currently, CCN3/NOV knockdown and overexpression experiments are on the way, and the results are likely to be presented on site.

\section{ROLE OF THE LOW-DENSITY LIPOPROTEIN RECEPTOR RELATED PROTEIN 1 (LRP1) IN CCN2/CONNECTIVE TISSUE GROWTH FACTOR (CTGF) PROTEIN TRANSPORTATION IN CHONDROCYTES}

Kazumi Kawata ${ }^{1}$, Satoshi Kubota ${ }^{1}$, Takanori Eguchi ${ }^{1}$, Eriko Aoyama ${ }^{2}$, Seiji Kondo ${ }^{1}$, Masaharu Takigawa ${ }^{1}$

${ }^{1}$ Department of Biochemistry and Molecular Dentistry, Okayama University Graduate School of Medicine, Dentistry and Pharmaceutical Sciences, and ${ }^{2}$ Biodental Research Center, Okayama University Dental School 
The low density lipoprotein receptor-related protein 1 (LRP1) has been known as an endocytic and signal transmission receptor. However, function of LRP1 in chondrocytes remained to be investigated. We formerly reported that LRP1 regulated the $\mathrm{WNT} / \beta$-catenin and protein kinase $\mathrm{C}$ signaling and repressed the hypertrophy during endochondral ossification, but its role of endocytosis in chondrocytes has not been understood. Moreover, CCN2/connective tissue growth factor (CTGF), which conducts endochondral ossification and cartilage repair, is one of the ligands of LRP1. In the present study, we have investigated the interaction of LRP1 and CCN2/CTGF during endocytotic traffic.

To address this issue, we performed RNAi to knockdown lrpl in condrocytes. The results showed that the amount of exogenous CCN2/ CTGF binding/incorporation was decreased in the lrpl knockdown cells. This result suggests LRP1 mediates the endocytotic events of $\mathrm{CCN} 2 / \mathrm{CTGF}$. Under this condition, ccn2/ctgf mRNA level was unchanged, whereas cell-associated CCN2/CTGF protein level was increased in lrpl knockdown chondrocytes. These results suggest LRP1 mediates also the exocytotic events of $\mathrm{CCN} 2 / \mathrm{CTGF}$. To examine transcytosis of CCN2/CTGF through chondrocytes, CCN2/CTGF, with/ without LRP1 antagonist, receptor-associated protein (RAP), was added to the cells on transwells, and the amount of transcytosed CCN2/CTGF that transferred to under chambers was analyzed. As a result, the amount of transcytosis of exogenous CCN2/CTGF was decreased by RAP. Moreover, under hypoxic condition, the levels of expression and production of LRP1 and the amount of transcytosis of CCN2/CTGF was increased, which was decreased by RAP.

These findings suggest that LRP1 mediates the transcytosis of CCN2/CTGF, which may be a critical event in cartilage biology.

\section{ROLE OF CCN2 IN EPITHELIAL TO MESENCHYMAL SWITCHING IN FIBROSIS}

Angela Tam ${ }^{1}$, Sonali Sonnylal ${ }^{2}$, Richard Stratton ${ }^{1}$, Andrew Leask ${ }^{3}$, Chris P Denton ${ }^{1}$, Jill Norman ${ }^{1}$, Benoit de Crombrugghe ${ }^{2}$, David J Abraham ${ }^{1}$, Xu Shi-wen ${ }^{1}$

${ }^{1}$ Division of Medicine, UCL Medical School, Royal Free Campus, London, UK; ${ }^{2}$ Department of Genetics, MD Anderson Cancer Center, University of Texas, Houston, TX, USA; and ${ }^{3} \mathrm{CIHR}$ Group in Skeletal Development and Remodeling, University of Western Ontario, London, ON, Canada

Background. Systemic Sclerosis (SSc) is a complex disorder of uncertain etiology characterised by progressive vascular and interstitial fibrosis. Recently, we found that the epidermal compartment of SSc skin exhibits abnormalities, taking on an activated phenotype reminiscent of that observed during the wound healing response. Fibroblast-epithelial cell interactions are believed to be important during normal tissue repair and aberrant cellular cues may underlie important aspects of fibrosis including epithelial to mesenchymal transition (EMT). Transgenic mice in which fibroblasts express a constitutively-active Transforming growth Factor $\beta$ (TGF $\beta$ ) type I receptor (ALK5) or which over-express Connective tissue growth factor (CTGF/CCN2), develop progressive tissue fibrosis most prominently in the skin and lung. Our in vivo data suggests that CCN2 over-expression causes stromal cell activation, as well as inducing pathological changes in the adjacent epithelium. Here, we focus on the role of CCN2 in epithelial to mesenchymal switching in the lung.
Materials and Method. Human and rat type II alveolar epithelial cell lines (A549 and T2) were grown in DMEM containing 10\% serum-starved over-night. Cells were stimulated with TGF $\beta(2 \mathrm{ng} / \mathrm{ml})$ in the presence and absence of siRNA specific for CCN2 or scrambled siRNA control for 24 hours. Markers of EMT such as decreased E-cadherin (the prototypic epithelial marker); increased CCN2, Snail, $\alpha$ SMA, collagen type I and fibronectin were examined by Western blotting.

Results. A549 and T2 epithelial cells exposed to TGF $\beta$ develop a mesenchymal-like morphology and express molecular markers associated with EMT (including Snail, CCN2, $\alpha$ SMA). In A549 cells, CCN2-specific siRNA dose-dependently suppressed TGF $\beta$ induced Snail and CCN2 protein expression towards basal levels. Transgenic mouse lung fibroblasts over-expressing CCN2 showed significantly higher levels of expression of extracellular matrix genes and proteins including collagen type I, fibronectin and also increased $\alpha$ SMA.

Summary. These data suggest that $\mathrm{CTGF} / \mathrm{CCN} 2$ is an important mediator of epithelial to mesenchymal switching in the lung. In SSc lung disease, over-expression of CTGF/CCN2 by stromal fibroblasts may drive the phenotypic switching increasing the number of profibrotic, matrix-producing mesenchymal cells.

\section{REGULATION OF CCN2 BY THE MIR-17-92 CLUSTER: IMPLICATIONS FOR TUMOR ANGIOGENESIS}

Jamie L. Fox, Michael Dews, Andrei Thomas-Tikhonenko

Department of Cell and Molecular Biology, University of Pennsylvania, Philadelphia, PA 19104; Department of Pathobiology, Children's Hospital of Philadelphia, Philadelphia, PA 19104

The role of CCN2 in angiogenesis is controversial: While some data suggests $\mathrm{CCN} 2$ promotes tumor neovascularization, additional evidence, including the presence of a thrombospondin type I repeat domain, implies an inhibitory role. Consistent with this idea, in Kras-transformed tp53-null murine colonocytes, c-Myc overexpression represses $\mathrm{CCN} 2$, which correlates with robust tumor vascularization and growth. Surprisingly, CCN2 downregulation by c-Myc occurs not via direct promoter binding, but through induction of the microRNA cluster miR-17-92. To assess the significance of this discovery for human cancers, we performed an unbiased metaanalysis of publicly available microarray data employing a set of 300 human tumor cell lines. Using this and other sets, we found an astonishingly strong negative correlation between expression levels of CCN2 and MIRH1, the primary transcript of miR-17-92. Then, using the human glioblastoma cell line A-172 as a model of solid human tumors with low-to-moderate miR-17-92 levels and corresponding moderate-to-high CCN2 expression, we analyzed the role of individual members of the miR-17-92 cluster in CCN2 downregulation. In murine colonocytes, miR-17-5p and miR-20a inhibit expression of TGFBR2 (TGF- $\beta$ receptor type II) and therefore dampen activation of some TGF- $\beta$ target genes, including CCN2. In contrast, in the A-172 model, only miR-18a inhibits basal CCN2 mRNA and protein levels. However, miR-18a also strongly dampens induction of $\mathrm{CCN} 2$ by TGF- $\beta$, perhaps via direct repression of Smad3. Currently, we are studying the functional effects of CCN2 overexpression or inhibition in in vivo models of solid human tumors to unequivocally establish its role in neovascularization and tumor growth. 